Notfall Rettungsmed 2010 · 13:621-634

DOI 10.1007/s10049-010-1371-2

Online publiziert: 16.11 .2010

(c) European Resuscitation Council 2010

H.-R. Arntz ${ }^{1}$ - L.L. Bossaert ${ }^{2} \cdot$ N. Danchin ${ }^{3} \cdot$ N. Nicolau ${ }^{4}$

${ }^{1}$ Medzinische Klinik 2, Kardiologie und Pulmologie,

Charité - Campus Benjamin Franklin, Berlin

${ }^{2}$ Abteilung für Intensivmedizin, Universität Antwerpen

${ }^{3}$ Abteilung für Koronarerkrankungen und kardiologische Intensivmedizin, Paris

${ }^{4}$ Coronary Care Unit, Athen

\title{
Initiales Management des akuten Koronarsyndroms
}

\section{Sektion 5 der Leitlinien zur Reanimation 2010 des European Resuscitation Council}

tienten mit Thoraxschmerzen) besteht darin, mithilfe wiederholter klinischer Biomarkeruntersuchungen und EKG-Registrierungen die Patienten zu identifizieren, die zur Durchführung invasiver Prozeduren aufgenommen werden müssen. Die Untersuchungen können Provokationstests und bei ausgewählten Patienten bildgebende Verfahren wie kardiale Computertomographie (KardioCT), Magnetresonanztomographie (MRT) etc. einschließen.

\section{Symptomatische Behandlung}

farkt-Akutes-Koronarsyndrom“" (Non-STEMI-ACS) wurde als gemeinsamer Begriff für NSTEMI und instabile Angina pectoris („unstable angina pectoris“, UAP) eingeführt, da die Differenzialdiagnose von Biomarkern abhängt, die erst nach mehreren Stunden nachweisbar sind. Dagegen beruhen Entscheidungen zur Therapie auf den klinischen Zeichen beim ersten Kontakt.

\section{"Chest pain units" und Regeln für die frühe Entlassung}

- Anamnese, klinische Untersuchung, Biomarker, EKG-Bild und RisikoScores identifizieren Patienten, die sicher entlassen werden können, nicht zuverlässig.

- Die Rolle von „chest pain units“

(CPU, spezialisierte Stationen für Pa-
- Nichtsteroidale antientzündliche Medikamente („nonsteroidal anti-inflammatory drugs“, NSAID) sollen nicht gegeben werden.

- Nitrate sollen nicht zu diagnostischen Zwecken eingesetzt werden.

- Sauerstoff soll nur Patienten mit Hypoxämie, Atemnot oder Lungenstauung verabreicht werden. Eine Hyperoxämie kann bei unkompliziertem Infarkt schädlich sein.

\section{Kausalbehandlung}

- Die Leitlinien für die Behandlung mit Acetylsalicylsäure (ASS) sind liberaler gestaltet worden. Acetylsalicylsäure kann durch Notfallzeugen mit oder ohne Anweisung des Leitstellendisponenten gegeben werden.
- Die Leitlinie für die Behandlung mit neuen Thrombozytenaggregationshemmern und Thrombinantagonisten bei Patienten mit STEMI und Non-STEMI-ACS wurde abhängig von der therapeutischen Strategie revidiert.

- Von der Gabe von Glykoprotein(Gp-)IIb/IIIa-Antagonisten vor Angiographie/perkutaner koronarer Intervention („percutaneous coronary intervention“, PCI) wird abgeraten.

\section{Reperfusionsstrategie bei ST-Hebungs-Infarkt}

— Die primäre PCI (PPCI) ist die bevorzugte Reperfusionsstrategie, sofern sie zeitgerecht durch ein erfahrenes Team durchgeführt werden kann.

- Der Rettungsdienst muss nicht das nächstgelegene Krankenhaus anfahren, sofern ein Herzkatheterlabor ohne zu große Verzögerung erreicht werden kann.

- Die akzeptable Verzögerung zwischen dem Beginn einer Fibrinolyse und einer ersten Ballondilatation variiert abhängig von Infarktlokalisation, Patientenalter und Symptomdauer erheblich zwischen etwa 45 und $180 \mathrm{~min}$.

Die ersten beiden Autoren haben zu gleichen Teilen zu diesem Manuskript beigetragen. 


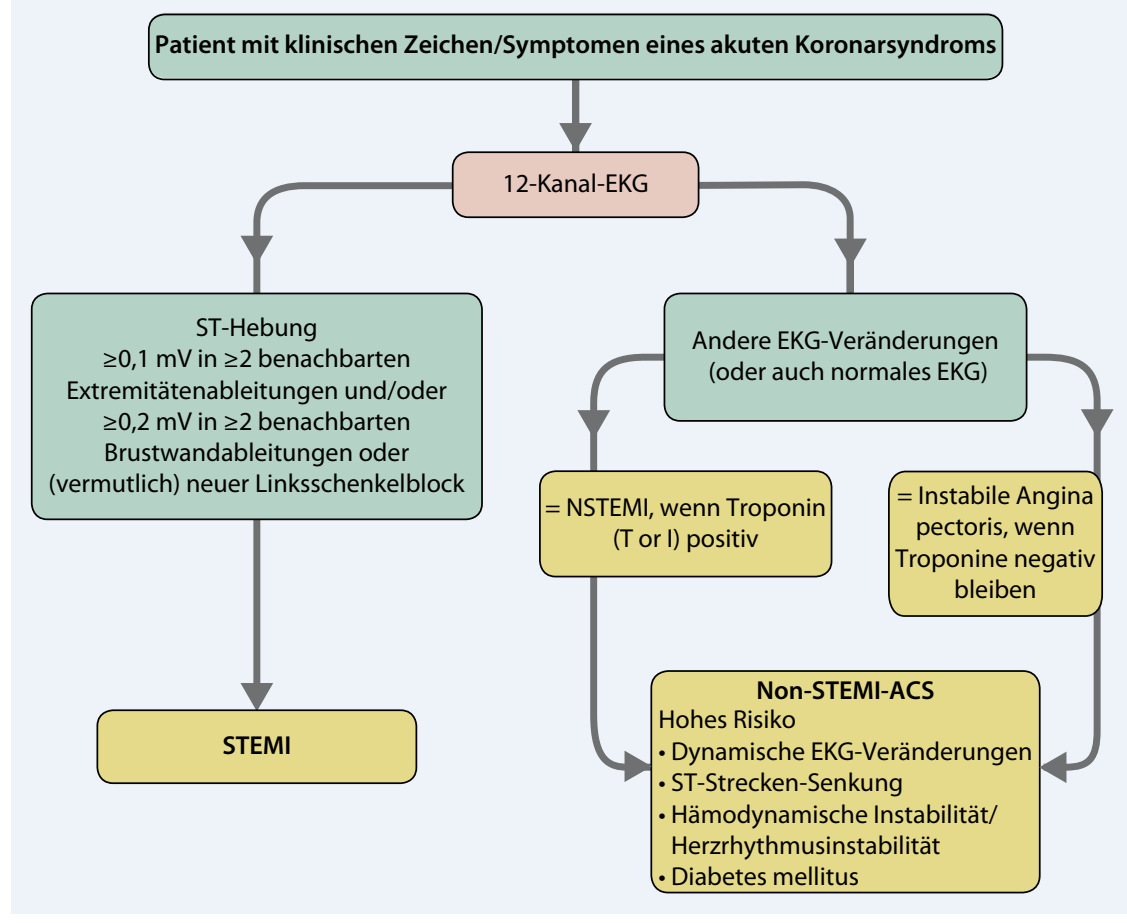

Abb. $1 \Delta$ Definitionen des ACS (ACS). STEMI ST-Hebungs-Infarkt, NSTEMI Nicht-ST-Hebungs-Infarkt

- Bei Versagen der Fibrinolyse soll eine „Rescue“-PCI durchgeführt werden.

- Von der Strategie einer Routine-PCI unmittelbar nach Fibrinolyse („facilitated PCI“) wird abgeraten.

- Patienten mit erfolgreicher Fibrinolyse in einem nicht-PCI-fähigen Krankenhaus sollen zur Angiographie und evtl. notwendigen PCI verlegt werden. Die PCI soll optimalerweise 6-24 h nach Fibrinolyse durchgeführt werden („pharmakoinvasives Vorgehen").

- Angiographie und, wenn nötig, PCI können bei Patienten nach erfolgreicher Reanimation („return of spontaneous circulation“, ROSC) sinnvoll sein. Sie können Teil eines standardisierten „Post-cardiac-arrest"-Protokolls sein.

- Um diese Ziele zu erreichen, ist es sinnvoll, Netzwerke unter Einschluss von Rettungsdienst, nicht-PCI-durchführenden Kliniken und PCI-durchführenden Kliniken zu bilden.

\section{Primäre und sekundäre Prävention}

- Die Empfehlung für die Gabe von $\beta$-Rezeptoren-Blockern wird eingeschränkt: Es gibt keine Evidenz für die routinemäßige intravenöse (i.v.-)
Gabe von $\beta$-Rezeptoren-Blockern außer in speziellen Situationen wie bei Tachyarrhythmien. Im Übrigen soll der $\beta$-Rezeptoren-Blocker oral in niedriger Dosierung begonnen werden, nachdem der Patient stabilisiert ist.

- Die Leitlinien zu der prophylaktischen Antiarrhythmikatherapie, dem Einsatz von Angiotensinkonversionsenzym- (ACE-)Hemmern bzw. Angiotensinrezeptorblocker (ARB) und Statinen bleiben unverändert.

\section{Einführung}

Die Inzidenz des akuten STEMI ist in vielen europäischen Ländern fallend [1], die Inzidenz des Non-STEMI-ACS jedoch ansteigend $[2,3]$. Obwohl die Krankenhaussterblichkeit bei STEMI durch die moderne Reperfusionstherapie und verbesserte Sekundärprophylaxe signifikant gesenkt werden konnte, ist die gesamte 28 -TageSterblichkeit praktisch unverändert geblieben, da zwei Drittel der Todesfälle vor Erreichen des Krankenhauses eintreten, meist durch letale Arrhythmien, die durch Ischämie getriggert sind [4]. Damit liegt die größte Chance, das Überleben einer ischämischen Attacke zu verbessern, darin, die Verzögerung zwischen Symptom- beginn und erstem professionellen medizinischen Kontakt zu verkürzen sowie eine gezielte Therapie bereits in der frühen prähospitalen Phase einzuleiten.

Der Begriff „ACS“ umfasst 3 Formen der akuten Manifestation der koronaren Herzkrankheit (KHK; - Abb. 1):

- ST-Strecken-Hebungs-Infarkt (STEMI),

- Nicht-ST-Hebungs-Infarkt (NSTEMI) und

- instabile Angina pectoris (UAP).

Der NSTEMI und die UAP werden üblicherweise unter dem Begriff Non-STEMI-ACS zusammengefasst. Die gemeinsame pathophysiologische Ursache des ACS ist eine rupturierte oder erodierte arteriosklerotische Plaque [5]. Elektrokardiographische Charakteristika (STHebung oder Fehlen einer ST-Hebung im EKG) grenzen den STEMI vom NonSTEMI-ACS ab. Letzteres Krankheitsbild kann mit ST-Strecken-Senkung, unspezifischen ST-Wellen-Veränderungen oder sogar mit einem normalen EKG einhergehen. Bei Fehlen einer ST-Hebung kann der NSTEMI durch den Anstieg der Konzentrationen kardialer Biomarker, speziell Troponin T oder Troponin I als spezifischste Marker myokardialer Zellnekrosen, im Plasma nachgewiesen werden.

Akute Koronarsyndrome sind die häufigste Ursache maligner Arrhythmien, die zum plötzlichen Herztod führen. Therapieziele sind die Beseitigung der akuten Lebensbedrohung, wie Kammerflimmern („ventricular fibrillation“, VF) oder extreme Brachykardien, der Erhalt der linksventrikulären Funktion und die Vorbeugung einer Herzinsuffizienz durch Minimierung des Ausmaßes des Myokardschadens. Die vorliegenden Leitlinien behandeln die ersten Stunden nach Beginn der Symptome. Die prähospitale Behandlung und die Initialbehandlung in der Notfallaufnahme können entsprechend lokaler Gegebenheiten, verfügbarer Ressourcen und Regelungen unterschiedlich sein. Die Daten, die die prähospitale Behandlung unterstützen, sind oft Extrapolationen von Studienergebnissen mit Initialbehandlung erst nach Krankenhausaufnahme. Es gibt wenige außerhalb des Krankenhauses durchgeführte Studien von hoher Qualität. Umfassende Leit- 
linien für die Diagnose und die Behandlung des ACS mit und ohne ST-StreckenHebung sind von der European Society of Cardiology (ESC) und dem American College of Cardiology (ACC)/American Heart Association (AHA) publiziert worden. Die vorliegenden Empfehlungen stimmen mit diesen Leitlinien überein [6, 7].

\section{Diagnose und Risikostratifizierung}

Da die Frühbehandlung den größten Nutzen bietet und die Myokardischämie der führende Auslöser des plötzlichen Herztodes ist, ist es wesentlich, dass die Öffentlichkeit mit den typischen Symptomen eines ACS vertraut ist. Bei einigen Patientengruppen jedoch ist die Wahrscheinlichkeit, dass sie bei Auftreten von ACSSymptomen medizinische Hilfe suchen, weniger wahrscheinlich. So wurden bei Frauen, älteren Menschen, Personen, die zu einer ethnischen oder rassischen Minderheit oder zu einer niedrigen sozioökonomischen Klasse gehören, sowie bei Patienten, die allein leben, signifikante Verzögerungen bis zum Beginn der Behandlung/Reperfusion berichtet [8].

Risikopatienten mit ihren Familien sollen fähig sein, die charakteristischen Symptome wie Brustschmerz, der in andere Regionen des Oberkörpers ausstrahlen kann und oft von weiteren Symptomen wie Luftnot, Schweißausbruch, Übelkeit oder Erbrechen und Synkopen begleitet wird, zu erkennen. Sie sollen die große Bedeutung einer frühen Aktivierung des Rettungsdienstes kennen und idealerweise in den Basismaßnahmen der Reanimation („basic life support“, BLS) geschult sein. Optimale Strategien, die Laien verschiedene Erscheinungsformen des ACS bewusst machen, und für eine Verbesserung der Kenntnis des ACS in der Risikobevölkerung, müssen noch entwickelt werden.

Darüber hinaus müssen Leitstellendisponenten im Rettungsdienst trainiert sein, die Symptome eines ACS zu erkennen und gezielte Fragen zu stellen. Wenn ein ACS-Verdacht besteht, soll ein Rettungsdienstteam alarmiert werden, das befähigt ist, erweiterte lebensrettende Maßnahmen („advanced life support“, ALS) einzuset- zen, die Diagnose zu stellen bzw. die Behandlung zu beginnen. Wegen der hohen Dringlichkeit der Revaskularisation bei STEMI und anderer Hochrisikopatienten können spezielle Systeme eingeführt werden, um die Erkennung von ST-StreckenHebungs-Infarkten zu verbessern und die Zeit bis zur Behandlung zu verkürzen.

Für das ACS sind Sensitivität, Spezifität und klinische Bedeutung verschiedener diagnostischer Strategien evaluiert worden. Sämtlich Information durch das klinische Erscheinungsbild, das EKG, Biomarkertests und bildgebende Verfahren sollen berücksichtigt werden, um die Diagnose zu sichern und gleichzeitig das Risiko einzuschätzen, sodass optimale Entscheidungen hinsichtlich der Patientenaufnahme und der Behandlung/Reperfusion getroffen werden können.

\section{Zeichen und Symptome}

Typischerweise tritt das ACS mit Symptomen wie ausstrahlender Brustschmerz, Luftnot und Schweißausbruch auf. Jedoch werden atypische und ungewöhnliche Erscheinungsformen bei älteren $\mathrm{Pa}$ tienten, Frauen und Diabetikern beobachtet $[9,10]$. Keines der Zeichen und Symptome des ACS kann allein Beleg für die Diagnose sein. Eine Abnahme des Brustschmerzes nach Nitroglyzeringabe kann fehlleitend sein; Nitroglyzerin wird als diagnostische Maßnahme nicht empfohlen [11]. Die Symptome können bei Patienten mit STEMI stärker sein und länger dauern; dies lässt aber die Unterscheidung zwischen Patienten mit STEMI und einem Non-STEMI-ACS nicht zu.

Die Vorgeschichte eines Patienten soll während des ersten Kontakts mit professionellen Helfern ausführlich erfasst werden. Sie kann die ersten Anhaltspunkte für das Vorhandensein eines ACS geben, Folgeuntersuchungen veranlassen und in Kombination mit anderen diagnostischen Testergebnissen bei der Triage und den therapeutischen Entscheidungen außerhalb des Krankenhauses sowie in der Notfallaufnahme helfen.

\section{2-Kanal-EKG}

Das 12-Kanal-EKG bildet die Schlüsseluntersuchung zur Beurteilung eines ACS.
Im Fall des STEMI zeigt es die Notwendigkeit der sofortigen Reperfusionstherapie (d. h. PPCI oder prähospitale Fibrinolyse). Wenn ein ACS-Verdacht besteht, soll ein 12-Kanal-EKG so früh wie möglich nach dem ersten Patientenkontakt abgeleitet und interpretiert werden, um eine frühe Diagnose und Triage zu erleichtern. Mithilfe des prähospitalen- oder Notfallaufnahme-EKG lassen sich nützliche diagnostische Informationen gewinnen, wenn das EKG von professionellen Anwendern interpretiert wird.

Die Registrierung eines 12-Kanal-EKG außerhalb des Krankenhauses ermöglicht vorab Informationen für die aufnehmende Institution und beschleunigt den Behandlungsprozess nach der Ankunft im Krankenhaus: In vielen Studien wurde die Zeit von der Krankenhausaufnahme bis zum Beginn der Reperfusionstherapie um 1060 min reduziert $[13,14]$. Geschultes Rettungsdienstpersonal (Notärzte, Rettungsassistenten und Pflegepersonal) kann einen STEMI - definiert durch ST-StreckenHebung $\geq 0,1 \mathrm{mV}$ in mindestens 2 zueinandergehörenden Extremitätenableitungen oder $\geq 0,2 \mathrm{mV}$ in mindestens 2 benachbarten Brustwandableitungen mit hoher Spezifität und Sensitivität identifizieren; dies ist mit der diagnostischen Genauigkeit im Krankenhaus vergleichbar $[15,16,17]$. Es ist daher sinnvoll, dass Rettungssanitäter und Pflegepersonal darin trainiert werden, einen STEMI ohne direkte ärztliche Rücksprache zu diagnostizieren, vorausgesetzt, dass sie einer strikten ärztlich überwachten Qualitätssicherung unterliegen.

Wenn eine EKG-Auswertung vor Ort nicht verfügbar ist, ist die computergestützte Interpretation $[18,19]$ oder die Funkübertragung des EKG sinnvoll. Registrierung und Übertragung von EKG in diagnostischer Qualität zum Krankenhaus dauern üblicherweise weniger als 5 min. Beim Einsatz bei Patienten mit Verdacht auf ein ACS kann die Computerinterpretation die Spezifität der STEMI-Erkennung v. a. in der EKG-Interpretation unerfahrener Kliniker verbessern. Der Nutzen der Computerinterpretation ist jedoch von der Qualität der Auswertung abhängig. Falsche Auswertungen können den unerfahrenen Beurteiler in die Irre führen. Demnach soll die computerassistierte EKG-Interpretation den erfahrenen 
Kliniker nicht ersetzen, sie kann aber als Hilfe zur Interpretation genutzt werden.

\section{Biomarker}

Beim Fehlen von ST-Strecken-Hebung im EKG charakterisieren eine verdächtige Vorgeschichte und erhöhte Konzentrationen von Biomarkern [Troponin T und Troponin I, Kreatinkinase (CK), KreatinkinaseTyp MB (CK-MB), Myoglobin] den NSTEMI und unterscheiden ihn damit vom STEMI und der UAP. Die Messung eines herzspezifischen Troponins ist vorzuziehen. Erhöhte Konzentrationen von Troponinen sind besonders hilfreich zur Identifikation von Patienten mit erhöhtem Risiko für ein ungünstiges Outcome [20]. Die Untersuchung kardialer Biomarker soll Teil der initialen Evaluierung aller Patienten der Notfallaufnahme sein, deren Symptome auf eine kardiale Ischämie verdächtig sind [21]. Die Zeitverlauf der Freisetzung von Biomarkern aus geschädigtem Myokard verhindert jedoch ihren Einsatz zur sicheren Diagnose eines Myokardinfarkts in den ersten 4-6 h nach Symptombeginn [22]. Bei Patienten, die sich innerhalb von $6 \mathrm{~h}$ nach Symptombeginn vorstellen und initial nichtpathologische Werte für kardiale Troponine aufweisen, sollen die Biomarker erneut 6-12 h nach Symptombeginn gemessen werden. Um die Bedeutung der gemessenen Biomarkerwerte optimal einzuordnen, sollen Kliniker die Sensitivität, Präzision und die Normwerte der verwendeten Tests kennen und mit der Freisetzungs- sowie Abbaukinetik der Marker vertraut sein. Hochsensitive (ultrasensitive) Assays für die kardialen Troponine stehen zur Verfügung. Sie ermöglichen die Sensitivität für die Diagnose eines Infarkts bei Patienten mit ischämieverdächtigen Symptomen zu erhöhen [23]. Wenn hochsensitive kardiale Troponin-Assays nicht verfügbar sind, kann eine Multimarkertestung unter Einschluss von CK-MB oder Myoglobin in Verbindung mit Troponinen infrage kommen, um die Sensitivität für eine Infarktdiagnose zu verbessern. Es gibt keine Daten, die den isolierten „Pointof-care“- (POCT-)Troponintest, um Patienten mit ischämieverdächtigen Symptomen zu beurteilen, als primäres Verfahren im prähospitalen Einsatz unterstützen [23]. In der Notfallaufnahme mag der Ge- brauch von POCT-Troponin-Tests hilfreich sein, um die Zeit bis zur Behandlung und die Dauer des Aufenthalts in der Notfallaufnahme zu verkürzen [24]. Bis die Resultate weiterer randomisierter kontrollierter Studien vorliegen, sollen andere SerumAssays nicht als Erstmaßnahme („first-line steps") für die Diagnose und Behandlung von Patienten mit ACS-Symptomen in Erwägung gezogen werden [25].

\section{Entscheidungsregeln für frühzeitige Entlassung}

Es hat eine Reihe von Versuchen gegeben, eine klinische Entscheidungsregel zu entwickeln, die in der Notfallaufnahme bei der Triage von Patienten mit Verdacht auf ein ACS hilfreich sein könnte. Dazu wurden Ergebnisse der Vorgeschichte des Patienten, körperlicher Untersuchungsbefund, serielle EKG und serielle Biomarkermessungen herangezogen. Keine dieser Regeln ist angemessen und geeignet, um Brustschmerzpatienten mit Verdacht auf ein ACS zu identifizieren, die sicher aus der Notfallaufnahme entlassen werden können [26]. Gleichermaßen sollen Scoring-Systeme zur Risikostratifizierung von Patienten mit ACS, die unter Krankenhausbedingungen evaluiert wurden [z. B. der Thrombolysis in Myocardial Infarction (TIMI) Score, der Global Registry of Acute Coronary Events (GRACE) Score, der Fast Revascularisation in Instability in Coronary Disease (FRISC) Score oder die Goldmann-Kriterien] nicht genutzt werden, um Patienten mit niedrigem Risiko, die aus der Notfallaufnahme entlassen werden können, zu identifizieren. Eine Subgruppe von Patienten im Alter unter 40 Jahren ohne klassische Beschwerden und signifikante Vorgeschichte mit Normalbefunden bei serieller Bestimmung von Biomarkern und 12-Kanal-EKG hat eine sehr geringe kurzfristige Ereigniswahrscheinlichkeit.

\section{Protokolle zur Beobachtung von Brustschmerzpatienten}

Bei Patienten mit Verdacht auf ein ACS reicht die Kombination einer leeren Anamnese und eines unauffälligen körperlichen Untersuchungsbefunds mit initial nichtpathologischem EKG und Biomarkerbefunden nicht aus, um ein ACS verlässlich auszuschließen. Deshalb ist es obligatorisch, den Patienten eine Zeit lang zu beobachten, um eine definitive Diagnose zu erzielen und therapeutische Entscheidungen zu treffen.

Protokolle für die Beobachtung von Brustschmerzpatienten sind Schnellsysteme zur Beurteilung von Patienten mit Verdacht auf ACS. Sie sollen generell die Vorgeschichte und die körperlichen Untersuchungsbefunde erfassen, gefolgt von einer Beobachtungsperiode, während der serielle EKG-Aufzeichnungen und Biomarkermessungen durchgeführt werden. Die Patientenbeurteilung soll entweder durch eine nichtinvasive Evaluierung der Koronaranatomie oder Provokationstests für Myokardischämie ergänzt werden, nachdem zunächst ein Infarkt ausgeschlossen wurde. Die Protokolle können genutzt werden, um die Treffsicherheit bei der Identifikation von den Patienten zu erhöhen, bei denen eine stationäre Aufnahme zur weiteren Diagnostik notwendig ist. Gleichzeitig erhalten sie die Patientensicherheit und reduzieren Aufenthaltsdauer sowie Kosten [27]. Bei Patienten, die mit Verdacht auf ein ACS die Notfallaufnahme aufsuchen, aber zunächst einen Normalbefund aufweisen, sind CPU möglicherweise eine sichere und effektive Strategie zur Patientenbeurteilung. Sie können empfohlen werden, um die Krankenhausaufenthaltsdauer zu verkürzen, die Zahl der Krankenhausaufnahmen und die Kosten zu reduzieren sowie die diagnostische Sicherheit und die Lebensqualität des Patienten zu verbessern [28]. Es gibt jedoch keine Daten, die beweisen, dass CPU oder Beobachtungsprotokolle unerwünschte kardiovaskuläre Ereignisse, insbesondere die Sterblichkeit von Patienten mit vermutetem ACS, vermindern.

\section{Bildgebende Verfahren}

Die Beurteilung von Patienten mit Verdacht auf ein ACS, aber nichtpatholgischem EKG und nichtpathologischem Ergebnis in der Bestimmung der kardialen Biomarker bleibt eine Herausforderung. Nichtinvasive bildgebende Verfahren (CT-Angiographie [29], MRT, Szintigraphie [30] und Echokardiographie [31]) wurden als Möglichkeit des Screening von Niedrigrisikopatienten geprüft. Ziel war 


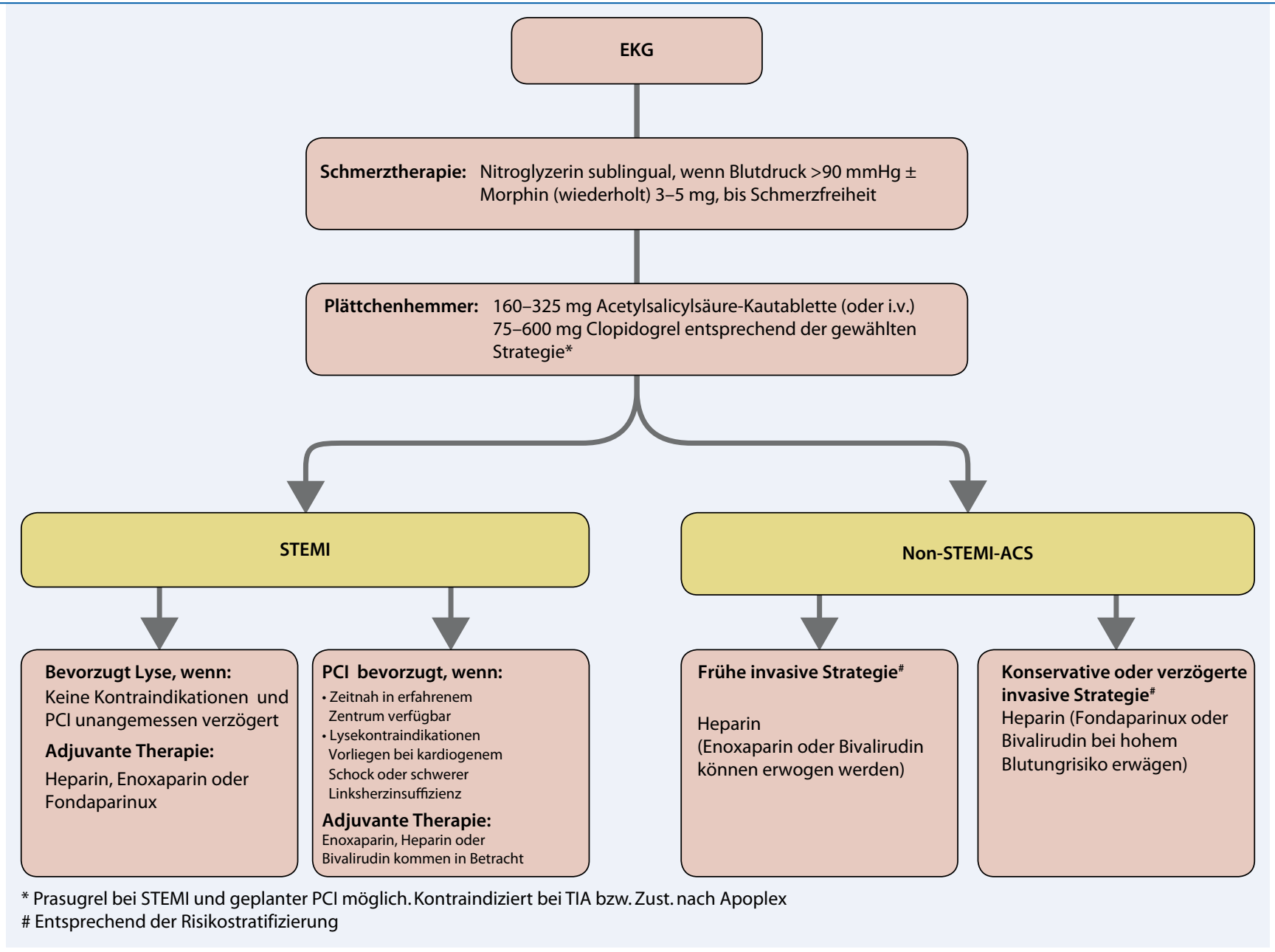

Abb. $2 \Delta$ Behandlungsalgorithmus für das akute Koronarsyndrom. Non-STEMI-ACS Nicht ST-Hebungs-Infarkt-Akutes-Koronarsyn-

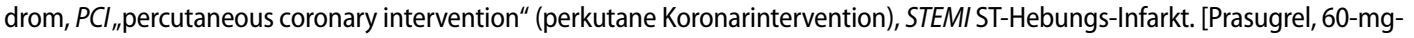
Initialdosis, kann bei Patienten mit STEMI als Alternative zu Clopidogrel und geplanter „, primary percutaneous coronary intervention" (PPCl) eingesetzt werden, sofern die Anamnese frei von Schlaganfall oder transitorischer ischämischer Attacke ist]

die Identifizierung von Subgruppen, die sicher nach Hause entlassen werden können. Obwohl es keine großen Multizenterstudie gibt, zeigen die existierenden Daten, dass diese diagnostischen Verfahren eine frühe und präzise Diagnose, eine Verkürzung der Aufenthaltsdauer sowie die Reduzierung der Kosten erlauben, ohne das Risiko kardialer Ereignisse zu erhöhen. Die Exposition gegenüber Strahlung und jodiertem Kontrastmittel soll jedoch beachtet werden, wenn eine kardiale CT bzw. eine Szintigraphie durchgeführt wird.

\section{Symptomatische Behandlung}

\section{Nitrate}

Nitroglyzerin ist eine effektive Behandlung des ischämischen Brustschmerzes und hat günstige hämodynamische Ef- fekte wie z. B. Dilatation venöser Kapazitätsgefäße, Erweiterung der Koronararterien und in geringerem Umfang der peripheren Arterien. Nitroglyzerin kann in Erwägung gezogen werden, wenn der systolische Blutdruck (SBD) über $90 \mathrm{~mm}$ Hg liegt und der Patient unter fortgesetztem ischämischem Brustschmerz leidet (• Abb. 2). Nitroglyzerin kann auch bei der Behandlung der akuten Lungenstauung nützlich sein. Nitrate sollen nicht bei Patienten mit Hypotension eingesetzt werden ( $\mathrm{SBD}<90 \mathrm{mmHg}$ ), speziell nicht bei gleichzeitiger Bradykardie und bei Patienten mit inferiorem Infarkt sowie Verdacht der rechtsventrikulären Beteiligung. Die Gabe von Nitraten unter diesen Bedingungen kann den Blutdruck und die kardiale Auswurfleistung senken.

\section{Analgesie}

Morphin ist das Analgetikum der Wahl für nitrorefraktären Schmerz, es hat außerdem beruhigende Wirkung, sodass Sedativa meist überflüssig sind. Da Morphin die venösen Kapazitätsgefäße dilatiert, kann es zusätzlich für Patienten mit pulmonaler Stauung vorteilhaft sein. Morphin soll mit Dosen von 3-5 mg i.v. begonnen und die Gabe in Abständen weniger Minuten wiederholt werden, bis der Patient schmerzfrei ist. Nichtsteroidale antientzündliche Medikamente (NSAID) sollen zur Analgesie wegen ihrer prothrombotischen Effekte nicht gegeben werden [32].

\section{Sauerstoff}

Die Messung der arteriellen Sauerstoffsättigung $\left(\mathrm{S}_{\mathrm{a}} \mathrm{O}_{2}\right)$ durch Pulsoxymetrie ist 
geeignet, um den Bedarf an zusätzlichem Sauerstoff festzustellen. Patienten benötigen keinen zusätzlichen Sauerstoff, solange sie nicht hypoxämisch sind. Einige der vorliegenden Daten deuten daraufhin, dass eine Sauerstoffgabe in hoher Konzentration für Patienten mit unkompliziertem Myokardinfarkt schädlich sein könnte $[33,34,35]$. Der Zielwert der $\mathrm{S}_{\mathrm{a}} \mathrm{O}_{2}$ soll 94-98\% sein bzw. 88-92\%, wenn das Risiko der Atemdepression mit $\mathrm{CO}_{2}$ - $\mathrm{Re}$ tention besteht [36].

\section{Kausale Behandlung}

\section{Thrombozytenaggre- gationshemmer}

Die Hemmung der Plättchenaggregation ist sowohl für die Initialbehandlung des ACS als auch für die Sekundärprävention von herausragender Bedeutung, da Plättchenaktivierung und Aggregation Schlüsselprozesse für die Auslösung eines ACS sind.

\section{Acetylsalicylsäure}

Große kontrollierte Studien führten zu einer reduzierten Sterblichkeit, wenn ASS (75-325 mg) bei Patienten mit ACS unter stationären Bedingungen gegeben wurde. Einige wenige Studien lassen eine verminderte Sterblichkeit vermuten, wenn ASS noch früher eingesetzt wird $[37,38]$. Deshalb soll ASS so früh wie möglich allen Patienten mit Verdacht auf ein ACS verabreicht werden, es sei denn, der Patient hat tatsächlich eine ASS-Allergie. Acetylsalicylsäure kann vom erstversorgenden professionellen Helfer, einem Notfallzeugen oder auf Anweisung des Leitstellendisponenten entsprechend lokaler Gepflogenheiten gegeben werden. Die Initialdosis von ASS-Kautabletten beträgt 160$325 \mathrm{mg}$. Andere Formen von ASS (löslich, i.v.-Präparation) sind wohl ebenso effektiv wie Kautabletten.

\section{Adenosindiphosphatre- zeptorantagonisten}

Thienopyridine (Clopidogrel, Prasugrel, Ticlopidin) inhibieren den Adenosindiphosphat- (ADP-)Rezeptor irreversibel, das Cyclopentyltriazolopyrimidin Ticagrelor reversibel. Die Wirkung addiert sich zur Plättchenaggregationshem- mung durch ASS. Im Gegensatz zu Clopidogrel ist der Metabolismus von Prasugrel und Ticagrelor unabhängig von der genetisch determinierten Variabilität des Arzneimittelstoffwechsels und der Wirkstoffaktivierung. Deshalb führen Prasugrel und Ticagrelor zu einer verlässlicheren und stärkeren Inhibition der Plättchenaggregation. Eine große randomisierte Studie, die Clopidogrel (Initialdosis $300 \mathrm{mg}$, gefolgt von $75 \mathrm{mg}$ täglich) mit Prasugrel (Initialdosis $60 \mathrm{mg}$, gefolgt von $10 \mathrm{mg}$ täglich) bei Patienten mit ACS untersuchte, führte $\mathrm{zu}$ weniger gravierenden wesentlichen bedrohlichen Koronarereignissen („major adverse cardiac events“, MACE) unter Prasugrel. Allerdings war die Blutungsrate unter Prasugrel höher. Das Blutungsrisiko war besonders erhöht bei $\mathrm{Pa}$ tienten, die weniger als $60 \mathrm{~kg}$ wogen und älter als 75 Jahre waren [39]. Eine signifikant erhöhte intrakranielle Blutungsrate unter Prasugrel wurde bei Patienten mit der Vorgeschichte einer transitorischen ischämischen Attacke (TIA) und/oder Schlaganfall beobachtet. In einer anderen Studie erwies sich Ticagrelor dem Clopidogrel hinsichtlich des Auftretens von wesentlichen bedrohlichen kardialen Ereignissen (MACE, [40]) überlegen.

\section{ADP-Rezeptor-Antagonisten bei Non- STEMI-ACS.}

Clopidogrel

Wenn Clopidogrel zusätzlich zu Heparin und ASS bei Hochrisikopatienten mit Non-STEMI-ACS gegeben wird, verbessert es das Outcome [41, 42]. Auch wenn es keine große Studie gibt, die die Vorbehandlung mit Clopidogrel - in einer 300mg- oder 6oo-mg-Initialdosis - mit einer periinterventionellen Gabe vergleicht, soll die Behandlung nicht bis zur Angiographie bzw. Intervention verzögert werden, da die höchsten Ereignisraten während der Frühphase des ACS beobachtet werden. Bei nichtselektierten Patienten mit KHK, die einer PCI unterzogen wurden, war die Vorbehandlung mit einer höheren Clopidogrelinitialdosis mit einem besseren Outcome verbunden [43]. Deshalb soll Clopidogrel allen Patienten mit NonSTEMI-ACS so früh wie möglich zusätzlich zu ASS und einem Antithrombin verabreicht werden. Ist ein konservatives Vorgehen geplant, soll die Initialdosis $300 \mathrm{mg}$ betragen; bei einer geplanten PCI-Strategie ist eine Initialdosis von $600 \mathrm{mg}$ möglicherweise günstiger.

\section{Prasugrel}

Prasugrel (6o-mg-Initialdosis) kann anstelle von Clopidogrel Patienten mit Hochrisiko-Non-STEMI-ACS und geplanter PCI während der Angiographie gegeben werden, sofern die Koronarstenosen sich als für die PCI geeignet erweisen. Die Kontraindikationen (Vorgeschichte mit TIA oder Schlaganfall) sowie die Nutzen-Risiko-Relation bei Patienten mit hohem Blutungsrisiko (Gewicht $<60 \mathrm{~kg}$, Alter $>75$ Jahre) sollen bedacht werden.

Ticagrelor $^{1}$

Ticagrelor (180 mg "loading dose“) kann anstelle von Clopidogrel Patienten mit Non-STEMI-ACS und geplanter invasiver Strategie verabreicht werden. Bisher liegen keine Daten zur Behandlung mit Prasugrel oder Ticagrelor in der Prähospitalphase oder Notfallaufnahme vor.

\section{ADP-Rezeptor-Antagonisten bei ST-He- bungs-Infarkt.}

Clopidogrel

Obwohl es keine große Studie zur Vorbehandlung von STEMI-Patienten mit Clopidogrel bei geplanter PCI gibt, ist eine solche Strategie wahrscheinlich vorteilhaft. Da die Thrombozyteninhibition mit einer höheren Dosis ausgeprägter ist, wird für Patienten mit STEMI und geplanter PCI so früh wie möglich eine Initialdosis von $600 \mathrm{mg}$ empfohlen. Zwei große randomisierte Studien haben Clopidogrel bei Patienten mit STEMI, die konservativ oder mit Fibrinolyse behandelt wurden, mit Placebo verglichen [44, 45]. Eine Studie untersuchte Patienten im Alter bis zu 75 Jahren, die mit Fibrinolyse, ASS, einem Antithrombin und einer Initialdosis von $300 \mathrm{mg}$ Clopidogrel behandelt wurden [45]. Die Behandlung mit Clopidogrel führte zu weniger verschlossenen infarktbezogenen Koronararterien bei der Angiographie und weniger Reinfarkten, ohne dass es zu einem erhöhten Blutungsrisiko kam. Die andere Studie untersuchte Patienten mit STEMI ohne obere Altersgrenze, die konservativ oder mit Fibrino-

\footnotetext{
1 Zum Zeitpunkt der Erstellung dieser Leitlinie war Ticagrelor nicht zugelassen.
} 
lyse behandelt werden sollen. In dieser Untersuchung führte die Gabe von Clopidogrel (keine initiale Aufladung, $75 \mathrm{mg}$ täglich) im Vergleich zu Placebo zu weniger Todesfällen und einer Verminderung des kombinierten Endpunkts aus Tod und Schlaganfall [44]. Aus diesem Grund sollen Patienten mit STEMI zusätzlich zu ASS und einem Antithrombin Clopidogrel erhalten (300-mg-Initialdosis bis zu einem Alter von 75 Jahren und $75 \mathrm{mg}$ Clopidogrel ohne Initialdosis im Alter über 75 Jahren).

Prasugrel

Prasugrel mit einer Initialdosis von 6o mg kann zusammen mit ASS und einem Antithrombin bei Patienten mit STEMI und geplanter PCI eingesetzt werden (Kontraindikation besteht bei Vorgeschichte mit TIA/Schlaganfall). Das Verhältnis des Blutungsrisikos zum Nutzen bei Patienten mit $<60 \mathrm{kgKG}$ oder einem Alter $>75$ Jahren soll berücksichtigt werden. Es gibt weder Daten zur prähospitalen Behandlung mit Prasugrel noch Daten zum Prasugreleinsatz im Zusammenhang mit einer Fibrinolyse.

Ticagrelor

Ticagrelor kann in einer Initialdosis von $180 \mathrm{mg}$ bei Patienten mit STEMI und geplanter PCI verabreicht werden. Es liegen keine Daten zur prähospitalen Behandlung mit Ticagrelor und keine Daten zu Ticagrelor beim Einsatz im Zusammenhang mit Fibrinolyse vor.

\section{Glykoprotein- (Gp-) \\ Ilb/Illa-Inhibitoren}

Der Glykoprotein- (Gp-)IIb/IIIa-Rezeptor ist das gemeinsame Endglied der Plättchenaggregation. Eptifibatid und Tirofiban führen zu einer reversiblen Inhibition, während Abciximab in einer irreversiblen Inhibition des Gp-IIb/IIIa-Rezeptors resultiert. Ältere Studien aus der „Vor-Stent-Ära“ unterstützen den Einsatz dieser Substanzklasse überwiegend [46, 47]. Neuere Studien dokumentieren zumeist ein neutrales oder sogar verschlechtertes Outcome $[48,49,50,51]$. In jedem Fall ereigneten sich Blutungen unter Behandlung mit Gp-IIb/IIIa-Rezeptor-Blocker bei mehr Patienten sowohl in positiven als auch in den neutralen oder negativen Studien. Die Datenlage zur Routinevorbehandlung mit Gp-IIb/IIIa-Rezep- toren bei Patienten mit STEMI oder NonSTEMI-ACS ist nicht ausreichend. Bei Hochrisikopatienten mit Non-STEMIACS ist die Vorbehandlung („Upstream“Behandlung) mit Eptifibatid oder Tirofiban vor geplanter PCI akzeptabel; dagegen soll Abciximab nur während der PCI gegeben werden $[47,52]$. Neuere Alternativen zur Thrombozytenaggregationshemmerbehandlung sollen wegen des erhöhten Blutungsrisikos mit Gp-IIb/IIIaRezeptoren zusammen mit Heparinen vorgezogen werden.

\section{Antithrombine}

Unfraktioniertes Heparin (UFH) ist ein indirekter Thrombininhibitor, der in Kombination mit ASS als Zusatz bei fibrinolytischer Therapie oder primärer PCI eingesetzt wird. Er ist daher ein wichtiger Teil der Behandlung der UAP und des ACS. Die Limitationen von UFH umfassen den schwer voraussagbaren antikoagulatorischen Effekt beim individuellen Patienten, die Notwendigkeit, es i.v. zu applizieren, und die Notwendigkeit, die aktivierte partielle Thromboplastinzeit (aPTT) zu kontrollieren. Darüber hinaus kann Heparin eine Thrombozytopenie induzieren. Seit der Publikation der Leitlinien 2005 zum ACS wurden große randomisierte Studien durchgeführt, die verschiedene alternative Antithrombine für die Behandlung von Patienten mit ACS untersucht haben. Im Vergleich mit UFH haben diese Alternativen eine spezifische Faktor-Xa-Aktivität [niedermolekulare Heparine („low-molecular-weight heparin“, LMWH), Fondaparinux] oder sind direkte Thrombininhibitoren (Bivalirudin). Bei diesen neueren Antithrombinen ist es i. Allg. nicht notwendig, das Gerinnungssystem zu überwachen, und es besteht ein geringeres Risiko für Thrombozytopenien.

\section{Antithrombine bei Non-STEMI-ACS}

Im Vergleich mit UFH reduziert Enoxaparin den kombinierten Endpunkt aus Sterblichkeit, Myokardinfarkt und die Notwendigkeit einer dringlichen Revaskularisation, wenn es innerhalb der ersten 24-36 h nach Symptombeginn bei Non-STEMIACS eingesetzt wird [53, 54]. Obwohl Enoxaparin mehr geringgradige Blutungen als UFH verursacht, ist die Inzidenz ernster Blutungen nicht erhöht.

Blutungen verschlechtern die Prognose von Patienten mit ACS [55]. Fondaparinux und Bivalirudin führen zu weniger Blutungen als UFH $[56,57,58,59]$. In den meisten Studien, die Patienten mit NonSTEMI-ACS untersuchen, wurden die UFH-Alternativen erst nach der Krankenhausaufnahme eingesetzt. Es mag daher nicht zulässig sein, die Ergebnisse dieser Studien auf die prähospitale Situation oder die Notfallaufnahme zu übertragen.

Für Patienten mit einem geplanten initial konservativen Vorgehen sind Fondaparinux und Enoxaparin sinnvolle Alternativen zu UFH. Die Datenlage zur Empfehlung anderer LMWH als Enoxaparin ist unzureichend. Für Patienten mit erhöhtem Blutungsrisiko soll Fondaparinux oder Bivalirudin in Betracht gezogen werden. Bei Patienten mit geplant invasivem Vorgehen sind Enoxaparin bzw. Bivalirudin sinnvolle Alternativen zu UFH. In einer Studie wurden Katheterthromben bei Patienten beobachtet, die sich einer PCI unterzogen und Fondaparinux erhalten hatten, - zusätzliches war UFH notwendig [56]. Da Enoxaparin und Fondaparinux bei Patienten mit Nierenschädigung akkumulieren können, ist eine Dosisanpassung erforderlich. Bivalirudin oder UFH sind Alternativen in dieser Situation. Das Blutungsrisiko kann beim Wechsel des Antikoagulans ansteigen. Daher soll die initial eingesetzte Substanz beibehalten werden, mit Ausnahme von Fondaparinux; hier ist die zusätzliche UFH-Gabe für Patienten bei der PCI notwendig.

\section{Antithrombine bei \\ ST-Hebungs-Infarkt}

\section{Patienten mit Fibrinolysebehandlung.}

\section{Enoxaparin}

Verschiedene randomisierte Studien an Patienten mit STEMI unter Fibrinolyse haben gezeigt, dass die zusätzliche Behandlung mit Enoxaparin anstatt UFH bessere klinische Ergebnisse nach sich zog (unabhängig von dem eingesetzten Fibrinolytikum), aber mit einer leicht erhöhten Blutungsrate bei älteren Patienten (>75 Jahre) und bei Patienten mit geringem Körpergewicht (<6o kgKG) einherging [61, 62, 63]. Reduzierte Dosen von Enoxaparin bei 
den älteren und leichten Patienten reduzierten die Blutungsrate bei gleich verbessertem Outcome [64]. Es ist darüber hinaus sinnvoll, Enoxaparin anstatt UFH für die prähospitale Behandlung einzusetzen. Dosierung von Enoxaparin: Bei Patienten $<75$ Jahre ist der Initialbolus $30 \mathrm{mg}$ i.v., gefolgt von $1 \mathrm{mg} / \mathrm{kgKG}$ s.c. alle $12 \mathrm{~h}$. (Die erste s.c.-Dosis soll kurz nach dem i.v.-Bolus verabreicht werden). Patienten $>75$ Jahre werden mit o,75 mg/kgKG s.c. alle $12 \mathrm{~h}$ ohne initiale i.v.-Dosis behandelt. Patienten mit bekannter Nierenfunktionsstörung (Kreatinin-Clearence $<30 \mathrm{ml} / \mathrm{min}$ ) können mit einer s.c.-Dosis von $1 \mathrm{mg} / \mathrm{kgKG}$ Enoxaparin einmal täglich behandelt werden oder sollen UFH erhalten. Die Datenlage für andere LMWH ist für eine Empfehlung unzureichend.

\section{Fondaparinux}

Verschiedene Studien haben die Überlegenheit oder ein unverändertes Outcome gezeigt, wenn Fondaparinux mit UFH als Additiv bei Fibrinolyse für STEMI-Patienten verglichen wurde [56]. Fondaparinux (initial 2,5 mg s.c., gefolgt von 2,5 mg s.c. täglich) kann speziell beim Einsatz nichtfibrinspezifischer Fibrinolytika (z. B. Streptokinase) bei Patienten mit einer Plasmakreatininkonzentration $<3 \mathrm{mg} / \mathrm{dl}$ $(250 \mu \mathrm{mol} / \mathrm{l})$ in Betracht gezogen werden.

\section{Bivalirudin}

Die Datenlage für die Empfehlung von Bivalirudin anstelle von UFH für STEMIPatienten, die mit einer Fibrinolyse behandelt werden, ist für eine Empfehlung unzureichend. Da das Blutungsrisiko bei einem Wechsel der Antikoagulanzien erhöht werden kann, soll die initiale Subs$\tan z$ beibehalten werden. Dies gilt mit der Ausnahme von Fondaparinux. Hier ist zusätzlich UFH notwendig, wenn ein invasives Vorgehen geplant ist.

Antithrombine für Patienten mit STEMI, die mit PPCI behandelt werden sollen. Es gibt kaum Studien bei Patienten mit STEMI und geplanter PCI zum Beginn der Antithrombinbehandlung in der Prähospitalphase oder Notfallaufnahme. Daher müssen Behandlungsempfehlungen für diese Situation aus klinischen Untersuchungen extrapoliert werden, solange spezifischere Ergebnisse laufender Studien noch nicht verfügbar sind.

\section{Enoxaparin}

Mehrere Register und kleinere Studien haben ein günstiges oder neutrales Outcome nachgewiesen, wenn Enoxaparin mit UFH bei der heute üblichen PPCI (breiter Einsatz von Thienopyridinen und/oder Gp-IIb/IIIa-Rezeptor-Blockern; $[65,66])$ eingesetzt wurde. Daher ist Enoxaparin eine sichere und effektive Alternative zu UFH. Die Datenlage dazu, andere LMWH als Enoxaparin für die PPCI bei STEMI zu empfehlen, ist unzureichend. Bei einem Wechsel von UFH zu Enoxaparin oder umgekehrt könnte es zu einem gesteigerten Blutungsrisiko kommen; dieser soll daher vermieden werden [6o]. Eine Dosisanpassung von Enoxaparin ist bei Patienten mit Niereninsuffizienz notwendig.

\section{Fondaparinux}

Beim Vergleich mit UFH führte Fondaparinux im Kontext einer PPCI zu vergleichbarem klinischen Outcome bei weniger Blutungen [56]. Jedoch erforderten Thrombusbildungen am Kathetermaterial die zusätzliche Behandlung mit UFH. Selbst wenn Fondaparinux das Blutungsrisiko im Vergleich mit UFH bei STEMIPatienten, die mit einer PCI behandelt werden, reduziert, wird der Einsatz beider Substanzen im Vergleich mit der alleinigen UFH-Gabe nicht empfohlen. Die Dosis von Fondaparinux muss bei Patienten mit Nierenfunktionsstörung angepasst werden.

\section{Bivalirudin}

Zwei große randomisierte Studien ergaben weniger Blutungen sowie eine Reduktion der Kurz- und Langzeitsterblichkeit, wenn Bivalirudin mit UFH und einem Gp-IIb/IIIa-Rezeptor-Blocker bei Patienten mit STEMI und geplanter PCI eingesetzt wurde $[67,68,69]$. Eine Reihe anderer Studien und Fallserien zeigte ebenfalls bessere oder neutrale Ergebnisse und weniger Blutungen, wenn Bivalirudin mit UFH verglichen wurde. Deshalb ist Bivalirudin eine sichere Alternative zu UFH. Allerdings wurde eine gering erhöhte StentThrombose-Rate innerhalb der ersten $24 \mathrm{~h}$ nach PCI beobachtet [67].

\section{Strategien und Versorgungssysteme}

Unterschiedliche systematische Strategien zur Steigerung der Qualität der prähospitalen Versorgung von Patienten mit ACS wurden untersucht. Diese Strategien zielen hauptsächlich daraufhin ab, Patienten mit Infarkt schnell zu identifizieren, um die Zeit bis zur Reperfusionstherapie zu verkürzen. Ebenso wurden Triagekriterien entwickelt, um Hochrisikopatienten mit Non-STEMI-ACS für den Transport zu Interventionszentren mit 24-h-/7-Tage-Interventionsbereitschaft auszuwählen. In diesem Zusammenhang ist eine Reihe spezifischer Entscheidungen während der Initialversorgung zu fällen, die neben den diagnostischen Basisschritten und der Beurteilung sowie Interpretation des 12-Kanal-EKG notwendig sind. Diese Entscheidung betreffen:

1. Die Reperfusionsstrategie bei Patienten mit STEMI, d. h. PPCI vs. (prä-)hospitale Fibrinolyse.

2. Die Umgehung näher gelegener Krankenhäuser ohne PCI-Möglichkeit und Maßnahmen, die die Zeit bis zur Intervention zu verkürzen, wenn die PPCI die gewählte Strategie ist.

3. Vorgehensweisen in speziellen Situationen z. B. für Patienten, die erfolgreich nach nichttraumatischem Herzstillstand reanimiert wurden, bzw. Patienten mit Schock oder Patienten mit Non-STEMI-ACS, die instabil sind oder Hinweise auf ein sehr hohes Risiko zeigen.

\section{Reperfusionsstrategie bei Patienten mit ST-Hebungs-Infarkt}

Die Reperfusionsstrategie bei Patienten mit STEMI ist der wichtigste Fortschritt in der Behandlung des Infarkts seit den letzten 25 Jahren. Bei Patienten mit STEMI und einer Symptomdauer $<12$ h soll die Reperfusion sobald wie möglich, unabhängig von der gewählten Methode, eingeleitet werden [7, 70, 71, 72]. Die Reperfusion kann durch Fibrinolyse, durch PCI oder durch eine Kombination beider Verfahren erreicht werden. Die Effizienz der Reperfusionstherapie ist stark von der Symptomdauer abhängig. Die Fibrinolyse ist speziell in den ersten 2-3 h nach Symp- 
tombeginn effektiv; die PPCI ist weniger zeitabhängig.

\section{Fibrinolyse}

Eine Metaanalyse aus 6 Studien, die 6434 Patienten umfasste, dokumentierte eine $17 \%$ ige relative Abnahme der Sterblichkeit, wenn Patienten mit prähospitaler Fibrinolyse statt mit intrahospitaler Lyse behandelt wurden [74]. Ein effektives und sicheres System für die prähospitale Lyse erfordert angemessene diagnostische und therapeutische Möglichkeiten für den STEMI und seine Komplikationen. Idealerweise soll die Möglichkeit bestehen, mit erfahrenen Krankenhausärzten in Verbindung zu treten (z. B. „emergency physicians" oder Kardiologen). Der wesentliche Zeitgewinn der prähospitalen Lyse bestand in einem Vorteil von $60 \mathrm{~min}$, und die Ergebnisse waren unabhängig von der Erfahrung des Anwenders. Daraus lässt sich schließen, dass die prähospitale Lysetherapie bei Patienten mit STEMI oder Symptomen eines ACS und (vermutlich) neuem Linksschenkelblock vorteilhaft ist. Die Fibrinolysebehandlung kann von trainierten Rettungssanitätern, Pflegepersonal oder Ärzten, die ein bewährtes Protokoll nutzen, sicher eingesetzt werden $[75,76,77,78,79,80]$. Die Effizienz ist innerhalb der ersten $3 \mathrm{~h}$ nach Symptombeginn am größten [74]. Patienten mit Symptomen eines ACS und EKG-Zeichen eines STEMI bzw. einem vermutlich neuen Linksschenkelblock oder Zeichen eines strikt posterioren Infarkts, die sich primär in der Notfallaufnahme vorstellen, sollen so bald wie möglich eine Fibrinolyse erhalten, wenn kein zeitnaher Zugang zur PPCI möglich ist.

Risiken der fibrinolytischen Therapie. Professionelle Helfer, die die fibrinolytische Therapie einsetzen, müssen mit den Kontraindikationen und Risiken vertraut sein (• Tab. 1). Patienten mit großen Infarkten (z. B. erkennbar an ausgeprägten EKG-Veränderungen) haben die größte Wahrscheinlichkeit, von einer fibrinolytischen Therapie zu profitieren. Der Nutzen der fibrinolytischen Therapie ist bei inferioren Infarkten weniger eindrucksvoll als bei anterioren Infarkten. Ältere Patienten weisen eine absolut höhere Mortalität auf. Der absolute Vorteil der fibrinolytischen Therapie ist aber ähnlich wie bei jüngeren Patienten. Patienten >75 Jahre tragen ein erhöhtes Risiko einer intrakraniellen Blutung durch Fibrinolyse. Daher ist der absolute Nutzen der Fibrinolyse durch diese Komplikation vermindert. Das Risiko einer intrakraniellen Blutung ist bei Patienten mit einem SBD von $>180$ mmHg erhöht. Dieses Maß an Hypertonie ist eine relative Kontraindikation zur Fibrinolyse. Das Risiko zur intrakraniellen Blutung ist auch von den eingesetzten Antithrombinen und der Thrombozytenaggregationshemmung abhängig.

\section{Primäre perkutane koronare Intervention}

Die Koronarangioplastie mit oder ohne Stent-Einlage ist zur bevorzugten Behandlung für Patienten mit STEMI geworden, da sie sich in mehreren Studien und Metaanalysen der Fibrinolyse bezüglich des kombinierten Endpunkts aus Tod, Schlaganfall und Reinfarkt als überlegen erwiesen hat $[81,82]$. Diese Vorteile wurden gefunden, wenn die PPCI durch eine erfahrene Person in einem Zentrum mit hohem Interventionsvolumen und nach begrenzter Verzögerung vom medizinischen Erstkontakt bis zur ersten Balloninflation möglich war [83]. Deshalb ist die PPCI, sofern sie in einem Zentrum mit hohem Interventionsvolumen kurz nach medizinischem Erstkontakt (FMC) durch einen erfahrenen Untersucher mit ausreichender Übung durchgeführt wird, die bevorzugte Behandlung, da sie die Morbidität und Mortalität im Vergleich zur sofortigen Thrombolyse vermindert.

\section{Fibrinolyse versus primäre perkutane koronare Intervention}

Der Einsatz der PPCI ist durch den $\mathrm{Zu}$ gang zu Katheterlaboren mit erfahrenen Untersuchern und die Verzögerung bis zur ersten Balloninflation begrenzt. Die Fibrinolyse ist eine überall verfügbare Reperfusionsstrategie. Beide Behandlungsstrategien sind gut etabliert und über die letzten Dekaden Themen großer randomisierter Multizenterstudien gewesen. Während dieser Zeit haben sich beide Behandlungsformen signifikant weiterentwickelt, und der Wirksamkeitsnachweis ist breit gestreut. In den randomisierten Studien, die die PPCI mit fibrinolytischer Thera-
Tab. 1 Kontraindikationen zur Fibri-

nolyse (entsprechend den Leitlinien der European Society of Cardiology)

\begin{tabular}{l} 
Absolute Kontraindikationen \\
Immer nach hämorrhagischem Schlaganfall \\
oder Schlaganfall unklarer Ätiologie \\
Ischämischer Schlaganfall in den letzten \\
6 Monaten \\
Schädigung des zentralen Nervensystems \\
oder Neoplasie \\
Kürzliches wesentliches Trauma/operativer \\
Eingriff/Kopfverletzung (innerhalb der letzen \\
3 Wochen) \\
\hline $\begin{array}{l}\text { Gastrointestinale Blutungen innerhalb } \\
\text { des letzten Monats }\end{array}$ \\
\hline Bekannte hämorrhagische Diathese \\
\hline Aortendissektion \\
\hline Relative Kontraindikationen \\
Transitorische ischämische Attacke (TIA) \\
in den letzten 6 Monaten \\
\hline Orale Antikoagulanzientherapie \\
Schwangerschaft innerhalb einer Woche \\
nach Entbindung \\
\hline Nichtkomprimierbare Punktionen \\
Traumatische Wiederbelebung \\
Therapierefraktäre Hypertension \\
(systolischer Blutdruck $>180$ mmHg) \\
Fortgeschrittene Lebererkrankung \\
Bakterielle Endokarditis \\
Aktives Magengeschwür \\
\hline
\end{tabular}

pie vergleichen, lag die typische Verzögerung von der Entscheidung zur PPCI oder zum Beginn der fibrinolytischen Therapie bei weniger als $60 \mathrm{~min}$. Verschiedene Berichte und Register, die die Fibrinolyse (einschließlich der prähospitalen Anwendung) mit der PPCI vergleichen, weisen einen Trend zum verbesserten Überleben auf, wenn die fibrinolytische Therapie innerhalb von $2 \mathrm{~h}$ nach Symptombeginn eingeleitet und mit einer „Rescue“- oder verzögerten PCI kombiniert wurde [84, 85, 86]. In Registern, die die Standardbedingungen realistischer darstellen, variierte die akzeptable Verzögerung durch PPCI (d.h. Diagnose bis Balloninflation minus Diagnose bis zur Injektion des Thrombolytikums), bei der eine Überlegenheit der PPCI gegenüber der fibrinolytischen Therapie nachweisbar war, erheblich. Die akzeptable Verzögerung schwankte zwischen 45 und über 180 min, abhängig von den Patientenbedingungen (d.h. Alter, Lokalisation des Infarkts und Symptomdauer; [87]). Darüber hinaus gibt es weni- 
ge Daten für die Überlegenheit der PPCI gegenüber der Fibrinolyse bei speziellen Subgruppen wie z. B. Patienten nach aortokoronarer Bypassoperation („coronary artery bypass grafting“, CABG) mit Niereninsuffizienz oder Diabetes [88, 89]. Die Zeitverzögerung bis zur PCI kann durch eine Verbesserung der Versorgungssysteme signifikant verkürzt werden [13, 90, 91, 92, 93], z. B. durch:

- prähospitale EKG-Registrierung

- EKG-Übertragung in das

Aufnahmekrankenhaus,

- direkte Telefonalarmierung des

Katheterlabors,

- Herstellung der Katheterlaborbereitschaft innerhalb $20 \mathrm{~min}$,

- laufende Vorhaltung eines interventionellen Kardiologen im Krankenhaus,

- Feedback-Routine über die tatsächlichen Zeitabläufe,

- Unterstützung durch Engagement leitender Personen und

- Ermutigung zu einem teambasierten Vorgehen.

Wenn die PPCI nicht innerhalb eines angemessenen Zeitfensters durchgeführt werden kann, soll unabhängig von der Notwendigkeit einer dringlichen Verlegung eine sofortige Fibrinolyse in Betracht gezogen werden, sofern keine Kontraindikationen bestehen. Für Patienten mit Kontraindikationen zur Fibrinolyse soll die PCI trotz der Verzögerung angestrebt und nicht gänzlich auf eine Reperfusionstherapie verzichtet werden. Für Patienten mit STEMI, die im kardiogenen Schock sind, ist die primäre PCI (oder koronare Bypassversorgung) die bevorzugte Reperfusionsbehandlung. Die Fibrinolyse soll nur dann erwogen werden, wenn zur PCI eine wesentliche Verzögerung eintritt.

\section{Triage und Interhospitaltransport zur primären perkutanen koronaren Intervention}

Das Risiko von Tod, Reinfarkt und Schlaganfall für Patienten mit STEMI ist reduziert, wenn sie rasch von Krankenhäusern der Grundversorgung zu Interventionszentren verlegt werden [82, 94, 95]. Es ist weniger klar, ob eine sofortige Fibrinolysetherapie (innerhalb oder außerhalb des Krankenhauses) oder die Ver- legung zur PPCI bei jüngeren Patienten mit einem Vorderwandinfarkt und einer kurzen Symptomdauer von weniger als 2$3 \mathrm{~h}$ besser ist [87]. Die Verlegung zur PP$\mathrm{CI}$ ist sinnvoll für Patienten, die sich mit einer Symptomdauer von mehr als 3, aber weniger als $12 \mathrm{~h}$ vorstellen, sofern sie rasch erfolgen werden kann.

\section{Kombination von Fibrinolyse und perkutane koronare Intervention}

Die Fibrinolyse und die PCI können in verschiedenen Kombinationen eingesetzt werden, um den koronaren Blutfluss und die myokardiale Perfusion wiederherzustellen. Es gibt mehrere Wege, in denen die beiden Behandlungen kombiniert werden können. Es fehlt an einheitlicher Nomenklatur, um die PCI unter diesen Vorgehensweisen zu beschreiben. „Facilitated PCI“ wird zur Beschreibung der PCI unmittelbar nach Fibrinolyse gebraucht. „Pharmakoinvasive“ Strategie bezeichnet eine PCI, die routinemäßig 3-24 h nach der Fibrinolyse durchgeführt wird. „Rescue-PCI“ ist als PCI, die wegen Lyseversagen vorgenommen wird (nachgewiesen durch weniger als 50\%ige Resolution der ST-SegmentHebung 60-90 min nach der Fibrinolysebehandlung) definiert. Die Strategien sind von einer Routine-PCI zu unterscheiden, bei der die Angiographie und Intervention einige Tage nach erfolgreicher Fibrinolyse durchgeführt wird. Verschiedene Studien und Metaanalysen zeigen ein schlechteres Outcome bei Routine-PCI sofort oder so früh wie möglich nach Fibrinolyse [48, 95]. Deshalb wird die routinemäßige Facilitated PCI nicht empfohlen, selbst wenn spezielle Subgruppen von Patienten von diesem Vorgehen möglicherweise profitieren [96]. Es ist sinnvoll, die Angiographie und Intervention, wenn notwendig, bei Patienten mit Lyseversagen, entsprechend klinischer Zeichen und/oder unzureichender ST-Segment-Resolution durchzuführen. Im Fall einer erfolgreichen Fibrinolyse (nachgewiesen durch klinische Zeichen) und einer ST-Segment-Resolution $>50 \%$ wurde mit um einige Stunden verzögerter Angiographie nach Fibrinolyse („pharmakoinvasives" Vorgehen) ein verbessertes Outcome nachgewiesen. Diese Strategie schließt eine frühzeitige Verlegung zur Angiographie und, wenn notwendig, PCI nach fibrinolytischer Therapie ein $[98,99]$.

\section{Spezielle Situationen}

\section{Kardiogener Schock}

Der kardiogene Schock (und in gewissem Umfang das schwere Linksherzversagen) ist eine wichtige Komplikationen des ACS; dieser hat eine Sterblichkeit von >50\%. Der kardiogene Schock bei STEMI ist keine Kontraindikation zur fibrinolytischen Therapie, aber die PCI ist die Behandlung der Wahl. Eine frühe Revaskularisation (d. h. PPCI oder PCI früh nach Fibrinolyse) ist bei Patienten indiziert, die einen Schock innerhalb $36 \mathrm{~h}$ nach Symptombeginn eines Infarkts entwickeln und bei denen eine Revaskularisation möglich ist [100]. Der Verdacht auf einen rechtsventrikulären Infarkt soll bei Patienten entstehen, die einen inferioren Infarkt hatten, klinisch einen Schock und einen unauffälligen Lungenauskulationsbefund aufweisen. Die ST-Hebung $\geq 1 \mathrm{~mm}$ in Ableitung $\mathrm{V} 4 \mathrm{r}$ ist ein nützlicher Indikator eines rechtsventrikulären Infarkts. Diese Patienten haben eine Krankenhaussterblichkeit von bis $\mathrm{zu} 30 \%$ und profitieren ausgeprägt von einer Reperfusionstherapie. Bei diesen Patienten sollen Nitrate und andere Vasodilatoren vermieden werden; die Hypotension soll mit i.v.-Flüssigkeitsgabe behandelt werden.

\section{Reperfusion nach erfolgreicher kardiopulmonaler Reanimation}

Die KHK ist die häufigste Ursache des prähospitalen Kreislaufstillstands. Viele dieser Patienten haben einen akuten Koronarverschluss mit Zeichen eines STEMI im EKG. Ein Kreislaufstillstand auf dem Boden einer ischämischen Herzerkrankung kann auch ohne diese Befunde eintreten. Verschiedene Fallserien haben demonstriert, dass eine Angiographie und, wenn notwendig, eine PCI bei Patienten mit ROSC nach Kreislaufstillstand möglich sind. Bei vielen Patienten können Koronararterienverschlüsse oder höhergradige Stenosen identifiziert und behandelt werden. Die Fibrinolyse mag eine Alternative für Patienten mit EKG-Zeichen eines STEMI sein [101]. Deshalb soll bei Patienten mit STEMI oder einem neuen Linksschenkelblock im EKG bei ROSC nach prähospitalem Kreislaufstillstand eine sofortige Angiographie und PCI oder Fibrinolyse überlegt werden $[102,103]$. 
Es ist sinnvoll, die sofortige Angiographie und PCI bei ausgewählten Patienten durchzuführen, auch beim Fehlen einer ST-Hebung im EKG oder Fehlen von klinischen Befunden wie z. B. Brustschmerz. Ferner ist es sinnvoll, die Reperfusionsbehandlung in ein standardisiertes Nachbehandlungskonzept nach Kreislaufstillstand als Teil einer Strategie zur Verbesserung des Outcome einzubeziehen [104]. Die Reperfusionsbehandlung soll andere therapeutische Strategien einschließlich der therapeutischen Hypothermie nicht verhindern.

\section{Primäre und sekundäre Prävention}

Präventive Interventionsmaßnahmen bei Patienten mit einem ACS sollen sofort nach Krankenhausaufnahme eingeleitet werden bzw., wenn schon eingeleitet, fortgesetzt werden. Präventivmaßnahmen verbessern die Prognose durch Verringerung gravierender unerwünschter kardialer Ereignisse. Die medikamentöse Prävention umfasst: $\beta$-Rezeptoren-Blocker, ACE-Hemmer/ARB und Statine sowie die Basisbehandlung mit ASS und, sofern indiziert, Thienopyridinen.

\section{$\beta$-Rezeptoren-Blocker}

Verschiedene Studien, die im Wesentlichen in der Präreperfusionsära durchgeführt wurden, zeigten eine verminderte Sterblichkeit, Reinfarktinzidenz und Herzrupturrate sowie auch eine geringere Inzidenz von VF und supraventrikulärer Arrhythmie bei Patienten, die früh mit einem $\beta$-Rezeptoren-Blocker behandelt wurden [105]. Eine i.v.- $\beta$-Blockade reduziert möglicherweise die Sterblichkeit bei Patienten, die einer primären PCI unterzogen werden und die nicht unter oralen Therapie mit $\beta$-Rezeptoren-Blockern stehen.

Studien zu $\beta$-Rezeptoren-Blockern sind sehr unterschiedlich bezüglich des Beginns der Behandlung. Es gibt kaum Daten zur prähospitalen Gabe oder in der Notfallaufnahme. Darüber hinaus zeigen neuere Studien ein erhöhtes Risiko eines kardiogenen Schocks bei Patienten mit STEMI, wenn auch die Rate schwerer Tachyarrhythmien durch die $\beta$-Blockade re- duziert war [106]. Es gibt keine Evidenz für die routinemäßige i.v.- $\beta$-Blockade in der prähospitalen oder frühen Notfallaufnahmesituation. $\beta$-Rezeptoren-Blocker mögen in speziellen Situationen indiziert sein, wie z. B. bei schwerer Hypertension oder Tachyarrhythmien ohne Kontraindikationen. Es ist sinnvoll, $\beta$ Rezeptoren-Blocker mit niedrigen oralen Dosen erst zu beginnen, wenn der Patient stabilisiert ist.

\section{Antiarrhythmika}

Es gibt keine Evidenz für den Einsatz einer antiarrhythmischen Prophylaxe bei ACS. Kammerflimmern verursacht die meisten frühen Todesfälle bei ACS. Die Inzidenz des VF ist in den ersten Stunden nach Symptombeginn am höchsten. Dies erklärt, warum zahlreiche Studien mit dem Ziel, den prophylaktischen Effekt antiarrhythmischer Therapien nachzuweisen, durchgeführt wurden [107]. Antiarrhythmische Medikamente (Lidocain, Magnesium, Disopyramid, Mexiletin, Verapamil, Sotalol) wurden auf ihre prophylaktischen Effekte beim ACS untersucht. Die Prophylaxe mit Lidocain reduzierte die Inzidenz von VF, könnte aber die Sterblichkeit steigern [108]. Die Routinebehandlung von Infarktpatienten mit Magnesium verbessert die Sterblichkeit nicht. Die Arrhythmieprophylaxe mit Disopyramid, Mexiletin, Verapamil oder anderen Antiarrhythmika innerhalb der ersten Stunden eines ACS verbessert die Sterblichkeit ebenfalls nicht. Deshalb wird keine Antiarrhythmikaprophylaxe empfohlen.

\section{Hemmer des Angiotensin- konversionsenzyms und Angiotensinrezeptorblocker}

Orale Hemmer des Angiotensinkonversionsenzyms (ACE-Inhibitoren) reduzieren die Sterblichkeit bei Patienten mit akutem Myokardinfarkt mit oder ohne frühe Reperfusionstherapie. Die günstigen Effekte sind am ausgeprägtesten bei Patienten mit Vorderwandinfarkt, Lungenstauung oder einer linksventrikulären Ejektionsfraktion $<40 \%$. Angiotensinkonversionsenzymhemmer sollen bei einem SBD $<100 \mathrm{mmHg}$ oder bei bekannten Kontraindikationen für diese Substanzen nicht gegeben werden. Ein Trend zu höherer Sterblichkeit wurde beobachtet, wenn eine i.v.-ACE-Inhibitor-Behandlung innerhalb der ersten $24 \mathrm{~h}$ nach Symptombeginn begonnen wurde. Deshalb soll innerhalb von $24 \mathrm{~h}$ nach Symptombeginn Patienten mit akutem Infarkt ein oraler ACEInhibitor verabreicht werden, unabhängig davon, ob eine frühe Reperfusionstherapie geplant ist. Dies gilt insbesondere für Patienten mit Vorderwandinfarkt, Lungenstauung oder einer linksventrikulären Ejektionsfraktion $<40 \%$. Intravenös applizierte ACE-Inhibitoren sollen innerhalb $24 \mathrm{~h}$ nach Symptombeginn nicht gegeben werden. Ein ARB soll Patienten gegeben werden, die ACE-Inhibitoren nicht vertragen $[109,110]$.

\section{Statine}

Statine reduzieren die Inzidenz wesentlicher unerwünschter kardiovaskulärer Ereignisse, wenn sie früh innerhalb der ersten Tage nach Beginn eines ACS gegeben werden $[111,112]$. Eine Statintherapie soll innerhalb von $24 \mathrm{~h}$ nach Beginn der Symptome eines ACS in Betracht gezogen werden, wenn keine Kontraindikationen vorliegen [Zielwert des „Low-density-lipoprotein“-Cholesterins $<80 \mathrm{mg} /$ $\mathrm{dl}, 2,1 \mathrm{mmol} / \mathrm{l})$. Wenn die Patienten bereits eine Statintherapie erhalten, soll diese nicht unterbrochen werden [113].

\section{Korrespondierender Übersetzer \\ Prof. Dr. H.R. Arntz \\ Medizinische Klinik II \\ Kardiologie und Pulmologie \\ Charité - Campus Benjamin Franklin \\ Hindenburgdamm 30 \\ 12200 Berlin \\ hans-richard.arntz@charite.de}

Interessenkonflikt. Der korrespondierende Übersetzer gibt an, dass kein Interessenkonflikt besteht. 


\section{Literatur}

1. Tunstall-Pedoe $H$, Vanuzzo D, Hobbs M et al (2000) Estimation of contribution of changes in coronary care to improving survival, event rates, and coronary heart disease mortality across the WHO MONICA Project populations. Lancet 355:688-700

2. Fox KA, Cokkinos DV, Deckers J et al (2000) The ENACT study: a pan-European survey of acute coronary syndromes. European Network for Acute Coronary Treatment. Eur Heart J 21:14401409

3. Goodman SG, Huang W, Yan AT et al (2009) The expanded Global Registry of Acute Coronary Events: baseline characteristics, management practices, and hospital outcomes of patients with acute coronary syndromes. Am Heart J 158:193-201 e1-e5

4. Lowel $H$, Meisinger $C$, Heier M et al (2002) Sex specific trends of sudden cardiac death and acute myocardial infarction: results of the population-based KORA/MONICA-Augsburg register 1985 to 1998. Dtsch Med Wochenschr 127:2311-2316

5. Thygesen K, Alpert JS, White HD (2007) Universal definition of myocardial infarction. Eur Heart 28:2525-2538

6. Van Werf F de, Bax J, Betriu A et al (2008) Management of acute myocardial infarction in patients presenting with persistent ST-segment elevation: the Task Force on the Management of STSegment Elevation Acute Myocardial Infarction of the European Society of Cardiology. Eur Heart J 29:2909-2945

7. Antman EM, Anbe DT, Armstrong PW et al (2004) ACC/AHA guidelines for the management of patients with ST-elevation myocardial infarctionexecutive summary: a report of the American College of Cardiology/American Heart Association Task Force on Practice Guidelines (Writing Committee to Revise the 1999 Guidelines for the Management of Patients with Acute Myocardial Infarction). Circulation 110:588-636

8. Khraim FM, Carey MG (2009) Predictors of prehospital delay among patients with acute myocardial infarction. Patient Educ Couns 75:155161

9. Douglas PS, Ginsburg GS (1996) The evaluation of chest pain in women. N Engl J Med 334:13111315

10. Solomon CG, Lee TH, Cook EF et al (1989) Comparison of clinical presentation of acute myocardial infarction in patients older than 65 years of age to younger patients: the Multicenter Chest Pain Study experience. Am J Cardiol 63:772-776

11. Henrikson CA, Howell EE, Bush DE et al (2003) Chest pain relief by nitroglycerin does not predict active coronary artery disease. Ann Intern Med 139:979-986

12. Ioannidis JP, Salem D, Chew PW, Lau J (2001) Accuracy and clinical effect of out-of-hospital electrocardiography in the diagnosis of acute cardiac ischemia: a meta-analysis. Ann Emerg Med 37:461-470

13. Terkelsen CJ, Lassen JF, Norgaard BL et al (2005) Reduction of treatment delay in patients with ST-elevation myocardial infarction: impact of pre-hospital diagnosis and direct referral to primary percutanous coronary intervention. Eur Heart J 26:770-777
14. Brainard AH, Raynovich W, Tandberg D, Bedrick EJ (2005) The prehospital 12-lead electrocardiogram's effect on time to initiation of reperfusion therapy: a systematic review and metaanalysis of existing literature. Am J Emerg Med 23:351-356

15. Swor R, Hegerberg S, McHugh-McNally A et al (2006) Prehospital 12-lead ECG: efficacy or effectiveness? Prehosp Emerg Care 10:374-377

16. Masoudi FA, Magid DJ, Vinson DR et al (2006) Implications of the failure to identify high-risk electrocardiogram findings for the quality of care of patients with acute myocardial infarction: results of the Emergency Department Quality in Myocardial Infarction (EDQMI) study. Circulation 114:1565-1571

17. Feldman JA, Brinsfield K, Bernard S et al (2005) Real-time paramedic compared with blinded physician identification of ST-segment elevation myocardial infarction: results of an observational study. Am J Emerg Med 23:443-448

18. Kudenchuk PJ, Ho MT, Weaver WD et al (1991) Accuracy of computer-interpreted electrocardiography in selecting patients for thrombolytic therapy. MITI Project Investigators. J Am Coll Cardiol 17:1486-1491

19. Dhruva VN, Abdelhadi SI, Anis A et al (2007) STSegment Analysis Using Wireless Technology in Acute Myocardial Infarction (STAT-MI) trial. J Am Coll Cardiol 50:509-513

20. Antman EM, Tanasijevic MJ, Thompson B et al (1996) Cardiac-specific troponin I levels to predict the risk of mortality in patients with acute coronary syndromes. N Engl J Med 335:13421349

21. Collinson PO, Gaze DC, Morris F et al (2006) Comparison of biomarker strategies for rapid rule out of myocardial infarction in the emergency department using ACC/ESC diagnostic criteria. Ann Clin Biochem 43:273-280

22. Schuchert A, Hamm C, Scholz J et al (1999) Prehospital testing for troponin $\mathrm{T}$ in patients with suspected acute myocardial infarction. Am Heart J 138:45-48

23. Keller T, Zeller T, Peetz D et al (2009) Sensitive troponin I assay in early diagnosis of acute myocardial infarction. N Engl J Med 361:868-877

24. Renaud B, Maison P, Ngako A et al (2008) Impact of point-of-care testing in the emergency department evaluation and treatment of patients with suspected acute coronary syndromes. Acad Emerg Med 15:216-224

25. Mitchell AM, Garvey JL, Kline JA (2006) Multimarker panel to rule out acute coronary syndromes in low-risk patients. Acad Emerg Med 13:803-806

26. Hess EP, Thiruganasambandamoorthy V, Wells GA et al (2008) Diagnostic accuracy of clinical prediction rules to exclude acute coronary syndrome in the emergency department setting: a systematic review. CJEM 10:373-382

27. Farkouh ME, Smars PA, Reeder GS et al (1998) A clinical trial of a chest-pain observation unit for patients with unstable angina. Chest Pain Evaluation in the Emergency Room (CHEER) Investigators. N Engl J Med 339:1882-1888

28. Ramakrishna G, Milavetz JJ, Zinsmeister AR et al (2005) Effect of exercise treadmill testing and stress imaging on the triage of patients with chest pain: CHEER substudy. Mayo Clin Proc 80:322-329
29. Goldstein JA, Gallagher MJ, O'Neill WW e al (2007) A randomized controlled trial of multi-slice coronary computed tomography for evaluation of acute chest pain. J Am Coll Cardiol 49:863871

30. Forberg JL, Hilmersson CE, Carlsson $\mathrm{M}$ et al (2009) Negative predictive value and potential cost savings of acute nuclear myocardial perfusion imaging in low risk patients with suspected acute coronary syndrome: a prospective single blinded study. BMC Emerg Med 9:12

31. Nucifora G, Badano LP, Sarraf-Zadegan N et al (2007) Comparison of early dobutamine stress echocardiography and exercise electrocardiographic testing for management of patients presenting to the emergency department with chest pain. Am J Cardiol 100:1068-1073

32. Kearney PM, Baigent C, Godwin J et al (2006) Do selective cyclooxygenase- 2 inhibitors and traditional non-steroidal anti-inflammatory drugs increase the risk of atherothrombosis? Meta-analysis of randomised trials. BMJ 332:1302-1308

33. Rawles JM, Kenmure AC (1976) Controlled trial of oxygen in uncomplicated myocardial infarction. Br Med Bull 1:1121-1123

34. Wijesinghe M, Perrin K, Ranchord A et al (2009) Routine use of oxygen in the treatment of myocardial infarction: systematic review. Heart 95:198-202

35. Cabello JB, Burls A, Emparanza Jl et al (2010) Oxygen therapy for acute myocardial infarction. Cochrane Database Syst Rev 6:CD007160

36. O'Driscoll BR, Howard LS, Davison AG (2008) BTS guideline for emergency oxygen use in adult patients. Thorax 63(Suppl 6):vi1-vi68

37. Freimark D, Matetzky S, Leor J et al (2002) Timing of aspirin administration as a determinant of survival of patients with acute myocardial infarction treated with thrombolysis. Am J Cardiol 89:381-385

38. Barbash IM, Freimark D, Gottlieb S et al (2002) Outcome of myocardial infarction in patients treated with aspirin is enhanced by pre-hospital administration. Cardiology 98:141-147

39. Wiviott SD, Braunwald E, McCabe $\mathrm{CH}$ et al (2007) Prasugrel versus clopidogrel in patients with acute coronary syndromes. N Engl J Med 357:2001-2015

40. Wallentin L, Becker RC, Budaj A et al (2009) Ticagrelor versus clopidogrel in patients with acute coronary syndromes. N Engl J Med 361:1045-1057

41. Yusuf S, Zhao F, Mehta SR et al (2001) Effects of clopidogrel in addition to aspirin in patients with acute coronary syndromes without ST-segment elevation. N Engl J Med 345:494-502

42. Mehta SR, Yusuf S, Peters RJ et al (2001) Effects of pretreatment with clopidogrel and aspirin followed by long-term therapy in patients undergoing percutaneous coronary intervention: the PCICURE study. Lancet 358:527-533

43. Steinhubl SR, Berger PB, Mann JT 3rd et al (2002) Early and sustained dual oral antiplatelet therapy following percutaneous coronary intervention: a randomized controlled trial. JAMA 288:2411-2420

44. Chen ZM, Jiang LX, Chen YP et al (2005) Addition of clopidogrel to aspirin in 45,852 patients with acute myocardial infarction: randomised placebo-controlled trial. Lancet 366:1607-1621 
45. Verheugt FW, Montalescot G, Sabatine MS et al (2007) Prehospital fibrinolysis with dual antiplatelet therapy in ST-elevation acute myocardial infarction: a substudy of the randomized double blind CLARITY-TIMI 28 trial. JThromb Thrombolysis 23:173-179

46. Bosch X, Marrugat J (2001) Platelet glycoprotein Ilb/llla blockers for percutaneous coronary revascularization, and unstable angina and nonST-segment elevation myocardial infarction. Cochrane Database Syst Rev CD002130

47. Boersma E, Harrington RA, Moliterno DJ et al (2002) Platelet glycoprotein Ilb/llla inhibitors in acute coronary syndromes: a meta-analysis of all major randomised clinical trials.[erratum appears in Lancet 2002 Jun 15;359(9323):2120]. Lancet 359:189-198

48. Keeley EC, Boura JA, Grines CL (2006) Comparison of primary and facilitated percutaneous coronary interventions for ST-elevation myocardial infarction: quantitative review of randomised trials. Lancet 367:579-588

49. Stone GW, Grines CL, Cox DA et al (2002) Comparison of angioplasty with stenting, with or without abciximab, in acute myocardial infarction. N Engl J Med 346:957-966

50. 't Hof AW van, Ernst N, Boer MJ de et al (2004) Facilitation of primary coronary angioplasty by early start of a glycoprotein $2 \mathrm{~b} / 3 \mathrm{a}$ inhibitor: results of the ongoing tirofiban in myocardial infarction evaluation (On-TIME) trial. Eur Heart J 25:837-846

51. Pannu R, Andraws R (2008) Effects of glycoprotein Ilb/llla inhibitors in patients undergoing percutaneous coronary intervention after pretreatment with clopidogrel: a meta-analysis of randomized trials. Crit Pathw Cardiol 7:5-10

52. De Luca G, Gibson M, Bellandi F et al (2008) Early Glycoprotein Ilb-Illa inhibitors in Primary angioplasty (EGYPT) cooperation. An individual patients' data meta-analysis. Heart

53. TIMI-11B Investigators, Antman EM, McCabe CH et al (1999) Enoxaparin prevents death and cardiac ischemic events in unstable angina/nonQ-wave myocardial infarction. Results of the thrombolysis in myocardial infarction (TIMI) 11B trial. Circulation 100:1593-1601

54. Cohen M, Demers C, Gurfinkel EP et al (1997) A comparison of low-molecular-weight heparin with unfractionated heparin for unstable coronary artery disease. Efficacy and Safety of Subcutaneous Enoxaparin in Non-Q-Wave Coronary Events Study Group. N Engl J Med 337:447-452

55. Moscucci M, Fox KA, Cannon CP et al (2003) Predictors of major bleeding in acute coronary syndromes: the Global Registry of Acute Coronary Events (GRACE). Eur Heart J 24:1815-1823

56. Yusuf S, Mehta SR, Chrolavicius S et al (2006) Comparison of fondaparinux and enoxaparin in acute coronary syndromes. N Engl J Med 354:1464-1476

57. Mehta SR, Boden WE, Eikelboom JW et al (2008) Antithrombotic therapy with fondaparinux in relation to interventional management strategy in patients with ST- and non-ST-segment elevation acute coronary syndromes: an individual patient-level combined analysis of the Fifth and Sixth Organization to Assess Strategies in Ischemic Syndromes (OASIS 5 and 6) randomized trials. Circulation 118:2038-2046
58. Lincoff AM, Bittl JA, Harrington RA et al (2003) Bivalirudin and provisional glycoprotein Ilb/llla blockade compared with heparin and planned glycoprotein Ilb/llla blockade during percutaneous coronary intervention: REPLACE-2 randomized trial. JAMA 289:853-863

59. Stone GW, McLaurin BT, Cox DA et al (2006) Bivalirudin for patients with acute coronary syndromes. N Engl J Med 355:2203-2216

60. Ferguson JJ, Califf RM, Antman EM et al (2004) Enoxaparin vs unfractionated heparin in highrisk patients with non-ST-segment elevation acute coronary syndromes managed with an intended early invasive strategy: primary results of the SYNERGY randomized trial. JAMA 292:45-54

61. (o A) (2001) Efficacy and safety of tenecteplase in combination with enoxaparin, abciximab, or unfractionated heparin: the ASSENT-3 randomised trial in acute myocardial infarction. Lancet 358:605-613

62. Eikelboom JW, Quinlan DJ, Mehta SR et al (2005) Unfractionated and low-molecular-weight heparin as adjuncts to thrombolysis in aspirin-treated patients with ST-elevation acute myocardial infarction: a meta-analysis of the randomized trials. Circulation 112:3855-3867

63. Wallentin L, Goldstein P, Armstrong PW et al (2003) Efficacy and safety of tenecteplase in combination with the low-molecular-weight heparin enoxaparin or unfractionated heparin in the prehospital setting: the Assessment of the Safety and Efficacy of a New Thrombolytic Regimen (ASSENT)-3 PLUS randomized trial in acute myocardial infarction. Circulation 108:135-142

64. Antman EM, Morrow DA, McCabe CH et al (2006) Enoxaparin versus unfractionated heparin with fibrinolysis for ST-elevation myocardial infarction. N Engl J Med 354:1477-1488

65. Zeymer U, Gitt A, Junger C et al (2008) Efficacy and safety of enoxaparin in unselected patients with ST-segment elevation myocardial infarction. Thromb Haemost 99:150-154

66. Zeymer U, Gitt A, Zahn R et al (2009) Efficacy and safety of enoxaparin in combination with and without GP IIb/Illa inhibitors in unselected patients with ST segment elevation myocardial infarction treated with primary percutaneous coronary intervention. Eurolntervention 4:524-528

67. White HD, Aylward PE, Frey MJ et al (1997) Randomized, double-blind comparison of hirulog versus heparin in patients receiving streptokinase and aspirin for acute myocardial infarction (HERO). Hirulog Early Reperfusion/Occlusion (HERO) Trial Investigators. Circulation 96:21552161

68. Stone GW, Witzenbichler B, Guagliumi G et al (2008) Bivalirudin during primary $\mathrm{PCl}$ in acute myocardial infarction. N Engl J Med 358:22182230

69. Madsen JK, Chevalier B, Darius H et al (2008) Ischaemic events and bleeding in patients undergoing percutaneous coronary intervention with concomitant bivalirudin treatment. Eurolntervention 3:610-616

70. Bassand JP, Hamm CW, Ardissino D et al (2007) Guidelines for the diagnosis and treatment of non-ST-segment elevation acute coronary syndromes. Eur Heart J 28:1598-1660
71. Anderson JL, Adams CD, Antman EM et al (2007) ACC/AHA 2007 guidelines for the management of patients with unstable angina/non ST-elevation myocardial infarction: a report of the American College of Cardiology/American Heart Association Task Force on Practice Guidelines (Writing Committee to Revise the 2002 Guidelines for the Management of Patients with Unstable Angina/Non ST-Elevation Myocardial Infarction): developed in collaboration with the American College of Emergency Physicians, the Society for Cardiovascular Angiography and Interventions, and the Society of Thoracic Surgeons: endorsed by the American Association of Cardiovascular and Pulmonary Rehabilitation and the Society for Academic Emergency Medicine. Circulation 116:e148-e304

72. Kushner FG, Hand M, Smith SC Jr et al (2010) 2009 Focused Updates: ACC/AHA Guidelines for the Management of Patients with ST-Elevation Myocardial Infarction (updating the 2004 Guideline and 2007 Focused Update) and ACC/AHA/ SCAI Guidelines on Percutaneous Coronary Intervention (updating the 2005 Guideline and 2007 Focused Update): a report of the American College of Cardiology Foundation/American Heart Association Task Force on Practice Guidelines. Circulation 120:2271-2306. Erratum in: Circulation. 121(12):e257. Dosage error in article text

73. Boersma E, Maas AC, Deckers JW, Simoons ML (1996) Early thrombolytic treatment in acute myocardial infarction: reappraisal of the golden hour. Lancet 348:771-775

74. Morrison L, Verbeek PR, McDonald AC et al (2000) Mortality and prehospital thrombolysis for acute myocardial infarction: A meta-analysis. JAMA 283:2686-2692

75. (о A) (1993) Prehospital thrombolytic therapy in patients with suspected acute myocardial infarction. The European Myocardial Infarction Project Group. N Engl J Med 329:383-389

76. Weaver WD, Cerqueira M, Hallstrom AP et al (1993) Prehospital-initiated vs hospital-initiated thrombolytic therapy. The Myocardial Infarction Triage and Intervention Trial. JAMA 270:12111216

77. (o A) (1992) Feasibility, safety, and efficacy of domiciliary thrombolysis by general practitioners: Grampian region early anistreplase trial. GREAT Group. BMJ 305:548-553

78. Welsh RC, Travers A, Senaratne M et al (2006) Feasibility and applicability of paramedic-based prehospital fibrinolysis in a large North American center. Am Heart J 152:1007-1014

79. Pedley DK, Bissett K, Connolly EM et al (2003) Prospective observational cohort study of time saved by prehospital thrombolysis for ST elevation myocardial infarction delivered by paramedics. BMJ 327:22-26

80. Grijseels EW, Bouten MJ, Lenderink T et al (1995) Pre-hospital thrombolytic therapy with either alteplase or streptokinase. Practical applications, complications and long-term results in 529 patients. Eur Heart J 16:1833-1888

81. Keeley EC, Boura JA, Grines CL (2003) Primary angioplasty versus intravenous thrombolytic therapy for acute myocardial infarction: a quantitative review of 23 randomised trials. Lancet 361:13-20 
82. Dalby M, Bouzamondo A, Lechat P, Montalescot G (2003) Transfer for primary angioplasty versus immediate thrombolysis in acute myocardial infarction: a meta-analysis. Circulation 108:18091814

83. Magid DJ, Calonge BN, Rumsfeld JS et al (2000) Relation between hospital primary angioplasty volume and mortality for patients with acute MI treated with primary angioplasty vs thrombolytic therapy. JAMA 284:3131-3138

84. Steg PG, Bonnefoy E, Chabaud S et al (2003) Impact of time to treatment on mortality after prehospital fibrinolysis or primary angioplasty: data from the CAPTIM randomized clinical trial. Circulation 108:2851-2856

85. Bonnefoy E, Steg PG, Boutitie F et al (2009) Comparison of primary angioplasty and pre-hospital fibrinolysis in acute myocardial infarction (CAPTIM) trial: a 5-year follow-up. Eur Heart J 30:1598-1606

86. Kalla K, Christ G, Karnik R et al (2006) Implementation of guidelines improves the standard of care: the Viennese registry on reperfusion strategies in ST-elevation myocardial infarction (Vienna STEMI registry). Circulation 113:2398-2405

87. Pinto DS, Kirtane AJ, Nallamothu BK et al (2006) Hospital delays in reperfusion for ST-elevation myocardial infarction: implications when selecting a reperfusion strategy. Circulation 114:2019

88. Madsen MM, Busk M, Sondergaard HM et al (2005) Does diabetes mellitus abolish the beneficial effect of primary coronary angioplasty on long-term risk of reinfarction after acute ST-segment elevation myocardial infarction compared with fibrinolysis? (A DANAMI-2 substudy). Am J Cardiol 96:1469-1475

89. Peterson LR, Chandra NC, French WJ et al (1999) Reperfusion therapy in patients with acute myocardial infarction and prior coronary artery bypass graft surgery (National Registry of Myocardial Infarction-2). Am J Cardiol 84:1287-1291

90. Kereiakes DJ, Gibler WB, Martin LH et al (1992) Relative importance of emergency medical system transport and the prehospital electrocardiogram on reducing hospital time delay to therapy for acute myocardial infarction: a preliminary report from the Cincinnati Heart Project. Am Heart J 123:835-840

91. Le May MR, So DY, Dionne R et al (2008) A citywide protocol for primary $\mathrm{PCl}$ in ST-segment elevation myocardial infarction. N Engl J Med 358:231-240

92. Gross BW, Dauterman KW, Moran MG et al (2007) An approach to shorten time to infarct artery patency in patients with ST-segment elevation myocardial infarction. Am J Cardiol 99:1360-1363

93. Bradley EH, Herrin J, Wang Y et al (2006) Strategies for reducing the door-to-balloon time in acute myocardial infarction. N Engl J Med 355:2308-2320

94. Widimsky P, Groch L, Zelizko M et al (2000) Multicentre randomized trial comparing transport to primary angioplasty vs immediate thrombolysis vs combined strategy for patients with acute myocardial infarction presenting to a community hospital without a catheterization laboratory. The PRAGUE study. Eur Heart J 21:823-831

95. (o A) (2006) Primary versus tenecteplase-facilitated percutaneous coronary intervention in patients with ST-segment elevation acute myocardial infarction (ASSENT-4 PCI): randomised trial. Lancet 367:569-578
96. Herrmann HC, Lu J, Brodie BR et al (2009) Benefit of facilitated percutaneous coronary intervention in high-risk ST-segment elevation myocardial infarction patients presenting to nonpercutaneous coronary intervention hospitals. JACC Cardiovasc Interv 2:917-924

97. Gershlick AH, Stephens-Lloyd A, Hughes $S$ et al (2005) Rescue angioplasty after failed thrombolytic therapy for acute myocardial infarction. $\mathrm{N}$ Engl J Med 353:2758-2768

98. Danchin N, Coste P, Ferrieres J et al (2008) Comparison of thrombolysis followed by broad use of percutaneous coronary intervention with primary percutaneous coronary intervention for STsegmentelevation acute myocardial infarction: data from the french registry on acute ST-elevation myocardial infarction (FAST-MI). Circulation 118:268-276

99. Cantor WJ, Fitchett D, Borgundvaag B et al (2009) Routine early angioplasty after fibrinolysis for acute myocardial infarction. N Engl J Med 360:2705-2718

100. Hochman JS, Sleeper LA, Webb JG et al (1999) Early revascularization in acute myocardial infarction complicated by cardiogenic shock. SHOCK Investigators. Should We Emergently Revascularize Occluded Coronaries for Cardiogenic Shock. N Engl J Med 341:625-634

101. Arntz HR, Wenzel V, Dissmann R et al (2008) Outof-hospital thrombolysis during cardiopulmonary resuscitation in patients with high likelihood of ST-elevation myocardial infarction. Resuscitation 76:180-184

102. Garot P, Lefevre T, Eltchaninoff H et al (2007) Sixmonth outcome of emergency percutaneous coronary intervention in resuscitated patients after cardiac arrest complicating ST-elevation myocardial infarction. Circulation 115:1354-1362

103. Spaulding CM, Joly LM, Rosenberg A et al (1997) Immediate coronary angiography in survivors of out-of-hospital cardiac arrest. N Engl J Med 336:1629-1633

104. Sunde K, Pytte M, Jacobsen D et al (2007) Implementation of a standardised treatment protocol for post resuscitation care after out-of-hospital cardiac arrest. Resuscitation 73:29-39

105. Yusuf S, Peto R, Lewis J et al (1985) Beta blockade during and after myocardial infarction: an overview of the randomized trials. Prog Cardiovasc Dis 27:335-371

106. Chen ZM, Pan HC, Chen YP et al (2005) Early intravenous then oral metoprolol in 45,852 patients with acute myocardial infarction: randomised placebo-controlled trial. Lancet 366:16221632

107. Teo KK, Yusuf S, Furberg CD (1993) Effects of prophylactic antiarrhythmic drug therapy in acute myocardial infarction. An overview of results from randomized controlled trials. JAMA 270:1589-1595

108. Hine LK, Laird N, Hewitt P, Chalmers TC (1989) Meta-analytic evidence against prophylactic use of lidocaine in acute myocardial infarction. Arch Intern Med 149:2694-2648

109. Swedberg K, Held P, Kjekshus J et al (1992) Effects of the early administration of enalapril on mortality in patients with acute myocardial infarction. Results of the Cooperative New Scandinavian Enalapril Survival Study II (CONSENSUS II). N Engl J Med 327:678-684
110. (o A) (1998) Indications for ACE inhibitors in the early treatment of acute myocardial infarction: systematic overview of individual data from 100,000 patients in randomized trials. ACE Inhibitor Myocardial Infarction Collaborative Group. Circulation 97:2202-2212

111. Patti G, Pasceri V, Colonna G et al (2007) Atorvastatin pretreatment improves outcomes in patients with acute coronary syndromes undergoing early percutaneous coronary intervention: results of the ARMYDA-ACS randomized trial. J Am Coll Cardiol 49:1272-1278

112. Hulten E, Jackson JL, Douglas K et al (2006) The effect of early, intensive statin therapy on acute coronary syndrome: a meta-analysis of randomized controlled trials. Arch Intern Med 166:1814-1821

113. Heeschen C, Hamm CW, Laufs U et al (2002) Withdrawal of statins increases event rates in patients with acute coronary syndromes. Circulation 105:1446-1452 